\title{
Building-to-grid flexibility: Modelling and assessment metrics for residential demand response from heat pump aggregations \\ DOI:
}

10.1016/j.apenergy.2018.10.058

\section{Document Version}

Accepted author manuscript

Link to publication record in Manchester Research Explorer

Citation for published version (APA):

Mancarella, P. (2019). Building-to-grid flexibility: Modelling and assessment metrics for residential demand response from heat pump aggregations. Applied Energy. https://doi.org/10.1016/j.apenergy.2018.10.058

\section{Published in:}

Applied Energy

\section{Citing this paper}

Please note that where the full-text provided on Manchester Research Explorer is the Author Accepted Manuscript or Proof version this may differ from the final Published version. If citing, it is advised that you check and use the publisher's definitive version.

\section{General rights}

Copyright and moral rights for the publications made accessible in the Research Explorer are retained by the authors and/or other copyright owners and it is a condition of accessing publications that users recognise and abide by the legal requirements associated with these rights.

\section{Takedown policy}

If you believe that this document breaches copyright please refer to the University of Manchester's Takedown Procedures [http://man.ac.uk/04Y6Bo] or contact uml.scholarlycommunications@manchester.ac.uk providing relevant details, so we can investigate your claim.

\section{OPEN ACCESS}




\title{
Building-To-Grid flexibility: modelling and assessment metrics for residential demand response from heat pump aggregations
}

\author{
Lingxi Zhang ${ }^{* 1,3}$, Nicholas Good ${ }^{2,3}$, and Pierluigi Mancarella ${ }^{3,4}$ \\ ${ }^{1}$ Envision Energy Limited, SOHO ZhongShan Plaza, 200051, Shanghai, P.R. China \\ ${ }^{2}$ Upside Energy Ltd, 61 Spear St, Manchester, M1 1DF, UK \\ ${ }^{3}$ The School of Electrical and Electronic Engineering, The University of Manchester, Ferranti Building, \\ Manchester, M13 9PL, UK \\ ${ }^{4}$ Department of Electrical and Electronic Engineering, The University of Melbourne, Melbourne, \\ Australia
}

lingxi.zhang@outlook.com,nickpgood@yahoo.co.uk,pierluigi.mancarella@unimelb.edu.au

*Corresponding author Tel: +8617521012692

\section{Highlights}

- Novel metrics are designed to quantify the building-to-grid demand response flexibility and investigate the behaviour of heating units following DR events.

- Power and energy paybacks following a heating load curtailment can vary from $0 \%$ to $50 \%$.

- Dwellings with high thermal inertia show a smaller payback magnitude but a longer payback period.

- Installing a hybrid heating system, reduces power and energy paybacks to less than $20 \%$ with a maximum comfort loss of $0.5^{\circ} \mathrm{C}$.

\begin{abstract}
Increased flexibility has been identified as a key requirement in future power systems. Much flexibility could be provided by energy vectors other than electricity. In particular heat may be a valuable source of flexibility, as electrification of space and water heating introduces highly flexible resources such as electric heat pumps. However, current methods for assessing aggregated demand side flexibility, particularly from residential buildings, may not be adequate given the variety of different grid services that flexibility may be used to provide to different stakeholders, and considering relevant comfort constraints. On these bases, in this work several metrics, relevant to different stakeholders, are introduced to quantify building-to-grid demand response flexibility from heat pump aggregations. Specific control algorithms for the aggregations are also proposed and tested through a multi-energy residential energy consumption tool. A number of case studies are carried out to demonstrate the value of the proposed metrics and algorithms, especially in relation to flexibility exploitation with long sustain times (e.g., reserve services), which can noticeably affect user comfort. Our results indicate that the payback behaviour of heating units following a demand response event can vary substantially with different types of dwellings. More specifically, the power payback is negligible in dwellings with high thermal inertia, while the increase of power magnitude and energy consumption can reach $10 \%$
\end{abstract}


and $50 \%$, respectively, in dwellings with low thermal inertia. The benefits from hybrid (electric + gas) heating, which can reduce energy payback and comfort loss, are also demonstrated. For instance, in a cluster of dwellings with low thermal inertia, the energy payback following a DR event is reduced from $50 \%$ to $20 \%$ and the maximum comfort loss of the participants is decreased from $1.6{ }^{\circ} \mathrm{C}$ to 0.5 ${ }^{\circ} \mathrm{C}$.

Key words: Flexibility, demand response, electric heat pump aggregation, high-resolution building modelling, hybrid heating

\section{Glossary}

$\begin{array}{ll}\text { ASHP } & \text { air source heat pump } \\ \text { BaU } & \text { business as usual } \\ \text { CHP } & \text { combined heat and power } \\ \text { COP } & \text { coefficient of performance } \\ \text { DR } & \text { demand response } \\ \text { DHW } & \text { domestic hot water } \\ \text { DNO } & \text { distribution network operator } \\ \text { DSO } & \text { distribution system operator } \\ \text { EHP } & \text { electric heat pump } \\ \text { HVAC } & \text { heating and ventilation air conditioning } \\ \text { NG } & \text { National Grid } \\ \text { STOR } & \text { short term operating reserve } \\ \text { SO } & \text { system operator } \\ \text { TNO } & \text { transmission network operator } \\ \text { UK } & \text { United Kingdom }\end{array}$

\section{Nomenclature}

\section{Indices}

$n, N \quad$ dwelling number, set of dwellings in cluster

$m, M \quad$ dwelling rank in selection algorithm, set of selected dwellings for DR application

$t, T \quad$ simulation time step, set of simulation time steps (minutes)

$w, W \quad$ window of ancillary service, set of ancillary service windows (hours)

\section{Variables}

$A D D$

average disruption duration (minutes)

$A P C$

average power contribution (kW per dwelling)

$c$

worse comfort level $\left({ }^{\circ} \mathrm{C}\right)$

CMR

constant to maximum ratio (\%)

$E P R$

energy payback ratio (\%) 
IDP impacted dwellings percentage (\%)

NETR

net energy transfer ratio (\%)

$O C_{n, t}$

occupant's comfort level $\left({ }^{\circ} \mathrm{C}\right)$

$O C F_{n, t}$

occupant's comfort flag ( 0 or 1 )

$O C V_{n}$

$P_{n, \bar{t}}^{E} B a U$

daily maximum comfort loss/gain $\left({ }^{\circ} \mathrm{C}\right)$

$P_{n-t}^{E_{-} D R}$

input electrical power of an EHP in a business as usual case (kW)

$P_{n, \bar{t}}^{G_{-} B a U}$

input electrical power of an EHP in a demand response case (kW)

$P_{n, \bar{t}}^{G-D R}$

input rating of gas boiler in a business as usual case $(\mathrm{kW})$

$\mathrm{PoCV}_{c}^{-}$

input rating of gas boiler in a demand response case (kW)

$\mathrm{PoCV}_{c}^{+}$

$P P R$

comfort loss probability distribution (\%)

comfort gain probability distribution (\%)

$r_{t}^{U P, P} ; r_{t}^{D N, P} ;$

power payback ratio (\%)

$r_{t}^{U P, F} ; r_{t}^{D N, F}$

average upward/downward reserve service capacity per EHP in a cluster with

Partial/Full service procurement mechanism (kW)

$R_{t}^{U P} ; R_{t}^{D N}$

upward/downward reserve service requirement $(\mathrm{kW})$

$t f_{t}^{U P} ; t f_{t}^{D N}$

maximum available upward/downward flexibility of a EHPs cluster ( $\mathrm{kW})$

$T_{n, t}^{i n_{n} B a U}$

indoor temperature of a dwelling in a business as usual case $\left({ }^{\circ} \mathrm{C}\right)$

$T_{n, t}^{i n_{-} D R}$

indoor temperature of a dwelling in a demand response case $\left({ }^{\circ} \mathrm{C}\right)$

$T_{n, t}^{i n^{\text {band }}}$

indoor temperature dead-band $\left({ }^{\circ} \mathrm{C}\right)$

$T_{n, t}^{i n^{T G}}$

indoor target temperature $\left({ }^{\circ} \mathrm{C}\right)$

$T_{n, t}^{\text {in }}$ setback

indoor temperature setback point $\left({ }^{\circ} \mathrm{C}\right)$

TECR

total energy change ratio (\%)

$u_{n, t}^{E_{-} B a U}$

commitment states of EHP in a business as usual case (0 or 1$)$

$u_{n, t}^{E-D R}$

commitment states of EHP in a demand response case (0 or 1$)$

$u_{n, t}^{G-B a U}$

commitment states of gas boiler in a business as usual case (0 or 1$)$

$u_{n, t}^{G-D R}$

commitment states of gas boiler in a demand response case ( 0 or 1$)$

\section{Introduction}

Due to the threat of global warming and climate change, many jurisdictions have set ambitious targets for energy conversion from renewable technologies. However, generation variability and uncertainty from several renewable technologies mean that more flexibility ${ }^{1}$ will be required to integrate these technologies successfully [1]. In a traditional power system, flexibility is contributed by flexible

\footnotetext{
${ }^{1}$ In this work we define flexibility as the increase/decrease in electrical power (compared to a business-as-usual baseline) that can be provided by reducing/increasing load, respectively.
} 
generators and large-scale storage (e.g., pumped hydro). Currently, with communication technologies advancing, significant amounts of flexibility may now be available from the demand-side, and in particular the residential sector, particularly given electrification of heating [2].

Multiple actors could benefit from this demand side flexibility. For example, system operators could be interested in the potential of residential resources (particularly electro-thermal thermostatically controlled loads) to provide various frequency control services, of which more will be required in the near future [3]. System services of interest include fast frequency response [4]/regulation services [5] and slower reserve products [6]. Besides services to the system operator, network operators may also need flexibility to address network congestion [7], or may consider flexibility from demand side to minimise the cost of network expansion [8]. Retailers can also benefit from using residential flexibility to bid on energy markets to maximise their profits [9].

Understanding the potential of DR from the residential sector and particularly buildings to provide "building-to-grid" flexibility required by various power system actors requires two steps, namely, design of appropriate metrics and quantification of those metrics. Metrics may simply relate to the power that can be shifted/curtailed/increased, such as discussed in [10-13]. In these examples the flexibility of specific types of load can be assessed using software with bottom-up methods. For instance, EnergyPlus is used to simulate the energy consumption of commercial buildings and apartments in [10]. In that work, sensitivity studies are carried out with different building parameters and target temperature ranges to demonstrate the numerical distribution of potential flexibility of single building blocks for DR applications. On the other hand, there are many general metrics for flexibility quantification. Examples of other appropriate metrics are the appliance flexibility index and acceptable delay time metrics, as shown in [11] and [12], respectively. These two metrics measure flexibility as the acceptable time shifting of appliances' operation. Moreover, since the random behaviour of occupants may affect the individual appliance's flexibility, it is also important to quantify the flexibility of loads at the aggregate level. Hence, in [13] a metric called flexibility index of aggregate load is introduced, which is used to indicate the probability of demand increase or decrease of a group of loads. A further metric denoted as percentage flexibility level, which determines the amount of flexible demand for DR applications, is also given in [13]. Recognising that provision of flexibility products with long sustain times (e.g., few hours) can have an adverse effect on user comfort, the comfort level satisfaction of occupants is also used as the metric to correspond to the DR capacity as shown in [14]. Following another approach, the comfort level is considered intact if the temperature indicator is within the pre-defined bounds [15].

Other publications have focused on the utilisation of flexibility for practical applications, such as renewable integration, network congestion and capacity support. In [16], the flexibility of generic electric heating units is used to increase the renewable penetration level at a microgrid scale, while a sensitivity study is performed with different occupancy profiles and weather patterns. The effectiveness of domestic DR based on real-time price signals has also been investigated, as shown in [17]. The flexibility of electric heating can also actively participate in ancillary service markets by means of a novel thermostat technology which takes into consideration the uncertainties from both demand and generation sides [18]. Case studies in the field can also provide valuable information. For example, a field trial undertaken in Belgium [19] found that potential upward and downward flexibilities per household are $65 \mathrm{~W}$ and $430 \mathrm{~W}$ respectively. Extrapolating this to the national level implies that domestic flexibility could equal $1.8 \%$ (upward) and $12.1 \%$ (downward) of installed generation capacity in the country. In a New York State study [20], it has been found that the system capability of integrating wind generation can increase to $5 \mathrm{GW}$, which is equivalent to a $33 \%$ wind penetration level in the grid of New York State, if the EHP penetration level reach $20 \%$. In the UK case [21], it has been found that system peak demand can be reduced by $7 \mathrm{GW}$ (equivalent to $9 \%$ of the peak) by 2050 with the utilisation of the flexibility from domestic appliances and EVs. Despite the breadth of current research, a common gap in the above literature [10-18] is the neglect of the impact 
of DR in the period after service provision. Where this impact is considered, such as in the high-level case studies [19-21], there is no quantitative analysis on the payback effect of DR. In addition, continuous operation of DR can lead to the saturation of flexibility and the synchronisation of appliances, so that the service provision can eventually become unsustainable. Further, the recovery of flexibility in the post-DR period can lead to power spikes and additional energy consumption due to the synchronisation of appliances state, which can subsequently challenge system security. This behaviour is of significant interest to network and system operators. Hence, a comprehensive set of metrics for assessment of residential DR is clearly missing.

In order to cover the gaps mentioned above, this paper presents a unified framework to assess the flexibility of current and future residential heating technologies and their performance in providing building-to-grid demand response. Specific contributions include:

1) Several general flexibility metrics which are specifically designed to assess the benefits and impact of building-to-grid DR activities to different stakeholders in both electricity and gas sectors. In addition, the potential comfort loss of occupants due to DR is specifically investigated.

2) Two control algorithms of Electric Heat Pump (EHP) operation are introduced which are used to determine the on/off states of aggregated EHPs in the provision of ancillary services.

3) Simulation and assessment of DR from residential EHP aggregation for services with long sustain times is carried out.

4) The performance of EHP-only and hybrid (electricity-gas) heating systems is compared in different DR applications.

The rest of the paper is organized as follows: In Section 2, specific metrics are presented to assess EHP and hybrid heating systems' performance on grid services applied to different stakeholders, alongside the implemented DR control mechanisms. In Section 3, an overview of a high-resolution (one-minute) residential energy consumption model is presented, which is used to simulate the operation of EHPs and the comfort level of occupants. In Section 4, a grid reserve service is selected and various case studies are carried out to investigate the effectiveness and impact of DR on technical and economic perspectives. Section 5 concludes the findings of this paper and future works.

\section{Methodology}

In this section, the metrics which are used to quantify the operational flexibility of EHPs are explained. Then, various service impact metrics, classified by the energy system actors for which they will be of use, are described. Lastly, two service provision algorithms ('Full' and 'Partial') for controlling the EHP aggregation are introduced, which may lead to different values of the metrics in practice. Note that the approach described here is specifically for an EHP heated domestic building (in case with a gas boiler back-up), but that the approach is generalizable to other arrangements.

\subsection{Flexibility metrics and quantification}

Before the introduction of the metrics designed for different stakeholders in DR activities, it is necessary to define the quantity of EHPs' flexibility, which can be deployed for services provision. The amount of flexibility (in kW) provided by EHPs depend on their capacity and operational level. In the numerical applications carried out here, for the sake of simplicity, we assume that EHPs modulates between two states (on-off), as many devices actually do. However, these EHPs will normally cycle within the dead-band settings of the thermostat [22], with an equivalent average power output over the time windows of interest similar to that of heat pumps that are inverter-driven and can thus adjust their output level more finely. While the specific numerical results may change slightly, the general conclusions and the utilization of the metric set developed should not be affected. 
The upward/downward flexibility is equal to the amount of EHPs aggregated electric power input that can be reduced/increased to a minimum/maximum operational level starting from the current level. The terms "upward" and "downward" used here are in analogy to the power system's upward and downward reserve services that are normally provided by conventional generators, for which "upward" is associated to ramping "up" and downward to ramping "down". The maximum available upward/downward flexibility $\left(t f_{t}^{U P / D N}\right)$ of a cluster with $N$ EHP units is calculated as the sum of the flexibility from each unit, as shown in (1) and (2). $P_{n, t}^{E}$ represents the input electrical power rating of the EHP, while the binary variable $u_{n, t}$ indicates the commitment states of the EHP. It should be mentioned that the calculated flexibility is the instant value based on the original operation schedule of the unit at each time step. It means that any disruption of the unit's operation schedule may subsequently alter its flexibility quantity in the following hours. If the flexibility of EHP is used for DR application more than once within a day, the original flexibility quantity can be changed after the first flexibility utilisation, as the EHP needs to increase or reduce its heat generation to compensate the heat loss or over-heating phenomenon. In this work, only one DR application per day is considered, as continuous DR application within a day may severely diminish the consumer's experience.

$$
\begin{aligned}
& \mathbb{t f}_{t}^{U P}=\sum_{n=1}^{N}\left(P_{n, t}^{E} \cdot u_{n, t}^{E}\right) \\
& \operatorname{tf}_{t}^{D N}=\sum_{n=1}^{N}\left[P_{n, t}^{E} \cdot\left(1-u_{n, t}^{E}\right)\right]
\end{aligned}
$$

\subsection{Metrics and relevance to stakeholders}

Several different parties can be stakeholders (e.g., sellers or buyers) in DR activities. These are: system operator (SO), transmission network operator (TNO), distribution network operator (DNO) (or, in the future, Distribution System Operator (DSO)), retailer, aggregator, and consumer. In this section, metrics are separately designed for each stakeholder to quantify the impact of service provision activities during deployment and/or in post-service periods. The variables in the DR and business as usual (BaU) cases are differentiated (i.e., the input power of EHP $\left(P_{n, \bar{t}}^{E_{-} B a U}\right.$ and $\left.P_{n, \bar{t}}^{E_{-} D R}\right)$ in order to compare the heating units (i.e., EHPs, gas boilers) operation profiles of two scenarios.

\subsubsection{DNO/DSO, TNO and SO}

Although DR is used to support power system operation, a side effect of DR applications may be compromise of system security if there is not proper management, as the operation of EHPs can be synchronised after the service period. In the case of an upward service (that is, in the presence of a system imbalance due to generation shortage), the EHP cluster could potentially even switch off to decrease the demand and reduce the system imbalance. However, this will lead to indoor temperature drop that will require additional "payback" power to re-establish the desired temperature after the service has been called off. With regards to a downward service (system imbalance with generation excess), standby EHPs are ramped up/turned on to increase the system electric demand, which will lead to overheating and higher than desired temperature. Based on the explanation above, the changes of peak power due to DR activity are crucial information to network operators. A metric called Power Payback Ratio $(P P R)$ is created to quantify the variation of peak power. The PPR metric is calculated as the ratio of the maximum electrical power of the cluster in the DR and BaU cases within a day, as shown in (3), which is calculated with the input power of EHP $\left(P_{n, \bar{t}}^{E_{-} B a U}\right.$ and $\left.P_{n, \bar{t}}^{E_{-} D R}\right)$ and the corresponding operating states $\left(u_{n, t}^{E_{-} B a U}\right.$ and $\left.u_{n, t}^{E_{-} D R}\right)$, in BaU and DR cases. This metric can inform, for example, the DNO and TNO to avoid potential network congestion, and allow the SO to schedule an appropriate generation capacity. As mentioned above, DR applications can lead to the synchronisation of the EHPs' operation and subsequently increase the peak of electricity consumption. Therefore, the coincidence factor can be another important and generic metric for network operators [23]. 
In ancillary services markets it is common to procure a specific service for a certain duration over a given time 'window'. This means that the level of flexibility provided (i.e., the kW value of ancillary service that is provided) must be guaranteed over such time window. In this case, the Constant to Maximum Ratio $(C M R)$ may be of interest. The $C M R$ metric is calculated with the average service capacity of an $\operatorname{EHP}\left(r_{n, t}^{U P / D N}\right)$ and the maximum available flexibility of an EHP cluster $\left(t f_{t}^{U P / D N}\right)$ as shown in (4). The calculation of average service capacity $\left(r_{n, t}^{U P / D N}\right)$ is given in the Section 2.3. The $C M R$ indicates the proportion of a cluster's flexibility that is not being exploited due to the requirement to provide the same amount of flexibility throughout the window.

$$
\begin{aligned}
P P R & =\frac{\max _{t \in T}\left[\sum_{n=1}^{N}\left(P_{n, t}^{E_{-} D R} \cdot u_{n, t}^{E_{-} D R}\right)\right]}{\max _{t \in T}\left[\sum_{n=1}^{N}\left(P_{n, t}^{E_{-} B a U} \cdot u_{n, t}^{E_{-} B a U}\right)\right]} \\
C M R & =\sum_{t \in T}\left(r_{t}^{U P / D N} \cdot N\right) \cdot \frac{1}{\sum_{t \in T} t f_{t}^{U P / D N}}
\end{aligned}
$$

\subsubsection{Retailer}

Retailers can also benefit from specific DR applications, as it can reduce the imbalance of a retailer's position by temporarily adjusting customer demand. However, the payback effect of DR application can also lead to further changes on energy consumption of customers following the end of service provision. This payback is caused by the synchronisation of appliance operation as explained in Section 2.2.1. Three energy quantity related metrics are introduced to assess the impact of DR on retailer's bidding strategy in energy markets. The first metric is the Energy Payback Ratio $(E P R)$, which is calculated as the payback energy consumption divided by the energy volume of deployed upward/downward flexibility. As shown in (5), EPR is calculated using the input power of EHP $\left(P_{n, \bar{t}}^{E_{-} B a U}\right.$ and $\left.P_{n, t}^{E_{-} D R}\right)$ and the corresponding operating states $\left(u_{n, t}^{E_{-} B a U}\right.$ and $\left.u_{n, t}^{E_{-} D R}\right)$ in BaU and DR cases, and the average service capacity of an EHP $\left(r_{t}^{U P / D N}\right)$. This metric can be used to inform retailers of the potential changes of electricity consumption following DR events, which should be considered in the retailer's bidding strategy. The Total Energy Change Ratio (TECR) is calculated as the ratio of the EHP cluster's electricity consumption in DR and BaU cases, which involve the input power of EHP $\left(P_{n, \bar{t}}^{E_{B} B a U}\right.$ and $\left.P_{n, t}^{E_{-} D R}\right)$ and the corresponding operating states $\left(u_{n, t}^{E_{-} B a U}\right.$ and $\left.u_{n, t}^{E_{-} D R}\right)$ in BaU and DR cases as shown in (6). This metric can be used to assess the overall increase or reduction of electricity consumption caused by service provision. The TECR metric is valuable to retailers because this information can inform their decision on how much to bid in energy markets and the potential revenue changes associated with the electricity consumption of customers. The third metric is designed for hybrid heating system with both gas and electricity consumptions. For a hybrid heating system with an auxiliary gas boiler, it is possible to use the gas boiler to maintain the heat supply without much comfort loss. Therefore, the ratio of the net changes of gas and electricity consumption (the Net Energy Transfer Ratio - NETR $)$ is calculated in (7) with the input rating $\left(P_{n, \bar{t}}^{G_{B} B a U}\right.$ and $\left.P_{n, \bar{t}}^{G D R}\right)$ and operating states $\left(u_{n, t}^{G_{-} B a U}\right.$ and $\left.u_{n, t}^{G_{-} D R}\right)$ of the gas boiler in BaU and DR cases, the input power of EHP $\left(P_{n, \bar{t}}^{E_{-} B a U}\right.$ and $\left.P_{n, \bar{t}}^{E_{-} D R}\right)$ and the corresponding operating states $\left(u_{n, t}^{E_{-} B a U}\right.$ and $\left.u_{n, t}^{E_{-} D R}\right)$ in BaU and DR cases. This metric is important to retailers because many retailers may supply several energy types rather than electricity only. Therefore, the potential changes of customer energy consumption on other energy vector may cause concerns to retailers. The consumer may also be interested in the TECR and NETR metrics, as they will inform the energy bill changes due to DR activities. 


$$
\begin{aligned}
& E P R=\frac{\sum_{n=1}^{N}\left(\sum_{t \in T} P_{n, t}^{E_{-} D R} \cdot u_{n, t}^{E_{-} D R}-P_{n, t}^{E_{-} B a U} \cdot u_{n, t}^{E_{-} B a U}\right)}{\sum_{t \in T} r_{t}^{U P I D N} \cdot N} \\
& T E C R=\left\{\frac{\sum_{n=1}^{N}\left[\sum_{t \in T} P_{n, t}^{E_{-} D R} \cdot u_{n, t}^{E_{-} D R}\right]}{\sum_{n=1}^{N}\left[\sum_{t \in T} P_{n, t}^{E_{-} B a U} \cdot u_{n, t}^{E_{-} B a U}\right]}-1\right\} \\
& N E T R=\frac{\sum_{n=1}^{N}\left[\sum_{t \in T}\left(P_{n, t}^{G_{-} D R} \cdot u_{n, t}^{G_{-} D R}-P_{n, t}^{G_{-} B a U} \cdot u_{n, t}^{G_{-} B a U}\right)\right]}{\left|\sum_{n=1}^{N}\left[\sum_{t \in T}\left(P_{n, t}^{E_{-} D R} \cdot u_{n, t}^{E_{-} D R}-P_{n, t}^{E_{-} B a U} \cdot u_{n, t}^{E_{-} B a U}\right)\right]\right|}
\end{aligned}
$$

\subsubsection{Aggregator}

The aggregator is responsible for extracting the flexibility from individual consumers and providing various services to DR buyers. The aggregator will make payments to the individual consumers based on the committed flexibility amount. To do this fairly the aggregator must consider the impact of DR activity on the dwelling's EHP operation. For a cluster of EHPs, the impact of DR activities can be summarised according to three factors: the Impacted Dwellings Percentage (IDP); the Average Disruption Duration $(A D D)$ and the Average Power Contribution $(A P C)$. The IDP metric indicates the percentage of the dwellings with a different duration of EHP activation compared with BaU case, as calculated in (8) with the operating states of EHPs $\left(u_{n, t}^{E_{-} B a U}\right.$ and $\left.u_{n, t}^{E_{-} D R}\right)$ in BaU and DR cases. This metric informs the aggregator of how many dwellings in the cluster should receive compensation due to deployment. The $A D D$ metric can be used to measure the average change of EHP activation time across the service deployment period, which is also calculated using the operating states of EHPs $\left(u_{n, t}^{E-B a U}\right.$ and $\left.u_{n, t}^{E_{-} D R}\right)$ in BaU and DR cases, and IDP metric as shown in (9). The APC metric is calculated in (10) using the input power of EHP $\left(P_{n, t}^{E_{-} B a U}\right.$ and $\left.P_{n, t}^{E_{-} D R}\right)$ and the corresponding operating states $\left(u_{n, t}^{E_{-} B a U}\right.$ and $\left.u_{n, t}^{E_{-} D R}\right)$ in BaU and DR cases, which determine the average service contribution from one dwelling with an EHP. The $A D D$ and $A P C$ metrics can help an aggregator to calculate the payment to individual participants.

$$
\begin{aligned}
& I D P=\sum_{n=1}^{N}\left[\sum_{t \in T}\left(u_{n, t}^{E_{-} B a U}-u_{n, t}^{E_{-} D R}\right) \neq 0\right] \cdot \frac{1}{N} \\
& A D D=\sum_{n=1}^{N}\left(\sum_{t \in T}\left(u_{n, t}^{E_{-} B a U}-u_{n, t}^{E_{-} D R}\right)\right) \cdot \frac{1}{I D P \cdot N} \\
& A P C=\frac{\sum_{n=1}^{N}\left[\sum_{n=1}^{N}\left(\sum_{t \in T}\left(P_{n, t}^{E_{-} B a U} \cdot u_{n, t}^{E_{-} B a U}-P_{n, t}^{E_{-} D R} \cdot u_{n, t}^{E_{-} D R}\right)\right)\right]}{\sum_{n=1}^{N}\left(\sum_{t \in T}\left(u_{n, t}^{E_{-} B a U}-u_{n, t}^{E_{-} D R}\right)\right)}
\end{aligned}
$$

\subsubsection{Consumer}

The consumer is the essential source of demand-side flexibility. During the service provision process, the direct impact to the consumer would be the variation of their living comfort. Therefore, a metric 
is required to properly assess the probability of a dwelling experiencing a certain level of comfort loss or gain following a service provision. A metric is defined here, which is called Probability of Comfort Variation $(P o C V)$. The first stage in the calculation of POCV is to define the Occupant's Comfort Flag $\left(O C F_{n, t}\right) . O C F_{n, t}$ indicates the validity of the comfort change measurement with default value of zero and it is set to 1 when discomfort is experienced. This allows two circumstances to be excluded:

- The indoor temperature $\left(T_{n, t}^{i n_{B} B a U}\right.$ and $\left.T_{n, t}^{i n_{n} D R}\right)$ is within the target temperature $\left(T_{n, t}^{i n^{T G}}\right)$ plus/minus dead-band $\left(T_{n, t}^{\text {in }}{ }^{\text {band }}\right)$ range

- The indoor temperature is dropping from set point to setback point $\left(T_{n, t}^{i^{\text {isetback }}}\right)$ during the transition from active occupancy to inactive occupancy period

The rest of the time steps should be considered as uncomfortable periods, as shown in (11). Then the occupant's comfort level $\left(O C_{n, t}^{B a U / D R}\right)$ in these specific periods for DR and BaU cases are calculated in (12). This measures the deviation of the indoor temperature $\left(T_{n, t}^{i n_{-} B a U}\right.$ and $\left.T_{n, t}^{i n_{-} D R}\right)$ from the target temperature range which is calculated with the target temperature $\left(T_{n, t}^{i T^{T G}}\right)$ plus/minus the dead-band $\left(T_{n, t}^{\text {in }{ }^{\text {band }}}\right)$. Afterwards, the daily maximum comfort loss/gain $\left(O C V_{n}\right)$ of $\mathrm{BaU}$ and DR cases are determined with occupant's comfort level $\left(O C_{n, t}^{B a U / D R}\right)$ as shown in (13). The negative value of $O C V_{n}$ represents the worse comfort level in the DR cases and vice versa for positive value, which means comfort level improvement. Then, $O C V_{n}$ of each dwelling in the cluster is processed through cumulative probability distribution. The PoCV metric needs to be divided into two parts, which are the comfort loss and gain. $\mathrm{PoCV}_{c}^{-}$indicates the probability of a household having a certain level of worse comfort experience (denoted as $c$ in ${ }^{\circ} \mathrm{C}$ ) following its flexibility deployment, and vice versa for $\mathrm{PoCV}_{c}^{+}$, which measures the probability of a comfort level gain. $\mathrm{PoCV}_{c}^{-}$and $\mathrm{PoCV}_{c}^{+}$are defined in (14) and (15) respectively.

$$
\begin{aligned}
& O C F_{n, t}^{B a U / D R}=\left\{\begin{array}{l}
\quad \text { if }\left(T_{n, t}^{i n^{T G}}-T^{i n^{\text {band }}}<=T_{n, t}^{i n_{-} B a U / D R}<=T_{n, t}^{i n^{T G}}+T^{i n^{\text {band }}}\right) \\
\|\left(T_{n, t}^{i n^{T G}}==T_{n, t}^{i n^{\text {selback }}} \& T_{n, t}^{i n_{-} B a U / D R}>T_{n, t}^{i n^{\text {selaback }}}+T^{i n^{\text {band }}}\right) \\
\quad \text { else }
\end{array}\right. \\
& O C_{n, t}^{\text {BaU/DR }}=\min \left\{\left|T_{n, t}^{i n_{B} B a U / D R}-\left(T_{n, t}^{i n^{T G}}+T^{i n^{\text {band }}}\right)\right|,\left|T_{n, t}^{i n_{-} B a U / D R}-\left(T_{n, t}^{i n^{T G}}-T^{i n^{\text {band }}}\right)\right|\right\} \cdot O C F_{n, t}^{B a U / D R} \\
& O C V_{n}=\max _{t \in T}\left\{O C_{n, t}^{B a U}\right\}-\max _{t \in T}\left\{O C_{n, t}^{D R}\right\} \\
& P o C V_{c}^{-}=\sum_{n=1}^{N}\left(O C V_{n}<=c\right) \cdot \frac{1}{N} \\
& P o C V_{c}^{+}=\sum_{n=1}^{N}\left(c<=O C V_{n}\right) \cdot \frac{1}{N}
\end{aligned}
$$

\subsubsection{Metrics summary}

Based on the above discussion, Table 1 summarizes all the metrics introduced in this paper. These metrics can be used as a generic method in assessing not only the flexibility of EHPs, but also any flexible domestic appliances. More importantly, the metrics cover the assessment from multiple perspectives, such as technical, economic and social ones. For the technical part, metrics are presented which are used to assess the variation of peak demand and the shift of energy consumption. This information is essential for system and network operators to take preventive measures in order to prepare for energy and power payback following DR activation or even to alleviate payback through rescheduling the domestic appliances operation. It also indicates the average disruption time, which 
can be used to inform the economic question of how to quantify incentives paid to the contributors of flexibility. From the social perspective, the maximum deviation of comfort level caused by DR application is presented in a probabilistic way, which can be used to inform practical inconvenience which flexibility providers may experience in exchange for incentives.

Table 1. The summary of metrics designed for different stakeholders

\begin{tabular}{lll}
\hline Stakeholders & Metric name & Related content \\
\hline \multirow{3}{*}{ Operators (system and network) } & Power payback ratio (PPR) & Electrical power \\
\cline { 2 - 3 } & Coincidence factor & Electrical power \\
\cline { 2 - 3 } & Constant to maximum ratio (CMR) & Electricity \\
\hline \multirow{3}{*}{ Retailer } & Energy payback ratio (EPR) & Electricity \\
\cline { 2 - 3 } & Total energy change ratio (TECR) & Electricity \\
\cline { 2 - 3 } & Net energy transfer ratio (NETR) & Gas and electricity \\
\hline \multirow{3}{*}{ Aggregator } & Impacted dwelling percentage (IDP) & Time \\
\cline { 2 - 3 } & Average disruption duration (ADD) & Time \\
\hline Consumer & Average power contribution (APC) & Electrical power \\
\hline
\end{tabular}

\subsection{Service provision mechanism}

As highlighted above, the magnitude of potential flexibility in individual dwellings is relatively small, in the range of few kilowatts. In addition, the flexibility of a single house with an EHP can vary significantly in a short period due to the random behaviour of individual households. Therefore, it is necessary to aggregate thousands of houses into one cluster to increase the diversity of profiles. In addition, the information regarding the forecast of these operational profiles needs to be passed to system or network operators in advance, who contract residential flexibility to provide ancillary services. When the service is called, the aggregator remotely controls the individual appliances (i.e., EHPs) based on the capacity and sustain time requirements of the service. Moreover, the metrics should be assessed by aggregator to inform the potential impact of DR applications to other stakeholders. In Section 1, it is mentioned that two control algorithms are separately designed for system ancillary services and alleviation of network congestion. With regard to ancillary service provision, it is possible that only part of the EHP cluster's flexibility can be used for service bidding. Taking the UK power system as an example, the provision of these services requires a large and constant volume of flexibility to be available at specific time windows ${ }^{2}$, but the potential flexibility of a cluster can vary across the day. Thus, this algorithm, which is designed to deliver a constant service capacity, is called "Partial" in the rest part of the paper. The maximum contract volume of Partial algorithm is the minimum available load reduction/increase from the EHPs during those windows. Another algorithm introduced here is focused on other DR products which require the cluster to offer the maximum flexibility to mitigate the issues in the system. The algorithm for this case is denoted as "Full" in this paper.

A flow chart of the service provision control is shown in Figure 1. An aggregator can use one of the two control algorithms depending on specific applications. If the Partial algorithm is chosen, a priority list of dwellings is required to determine the switching on/off order of the EHPs. The list is created by comparing a specific indicator, such as indoor temperature. In the upward service case, the EHP of dwellings with higher indoor temperature $\left(T_{n, t}^{i n}\right)$ should be turned off first in order to minimise the impact of service operation on the occupant's comfort level and load diversity [5], and vice versa for

\footnotetext{
${ }^{2}$ Taking the short term operating reserve (STOR) as an example, the service windows are from 07:00 to $13: 00$ and $16: 00$ to $21: 00$ at half hour interval [36].
} 
the downward service case. After the EHP operation schedules of first $M$ dwellings are altered, whose total flexibility equals to the service requirement $\left(R_{t}^{U P / D N}\right)$, the control is looped again for next time step. With regard to the Full control mechanism, the instant flexibility of one cluster would be fully utilised, therefore all EHPs in this cluster are completely turned off/on for upward/downward service.

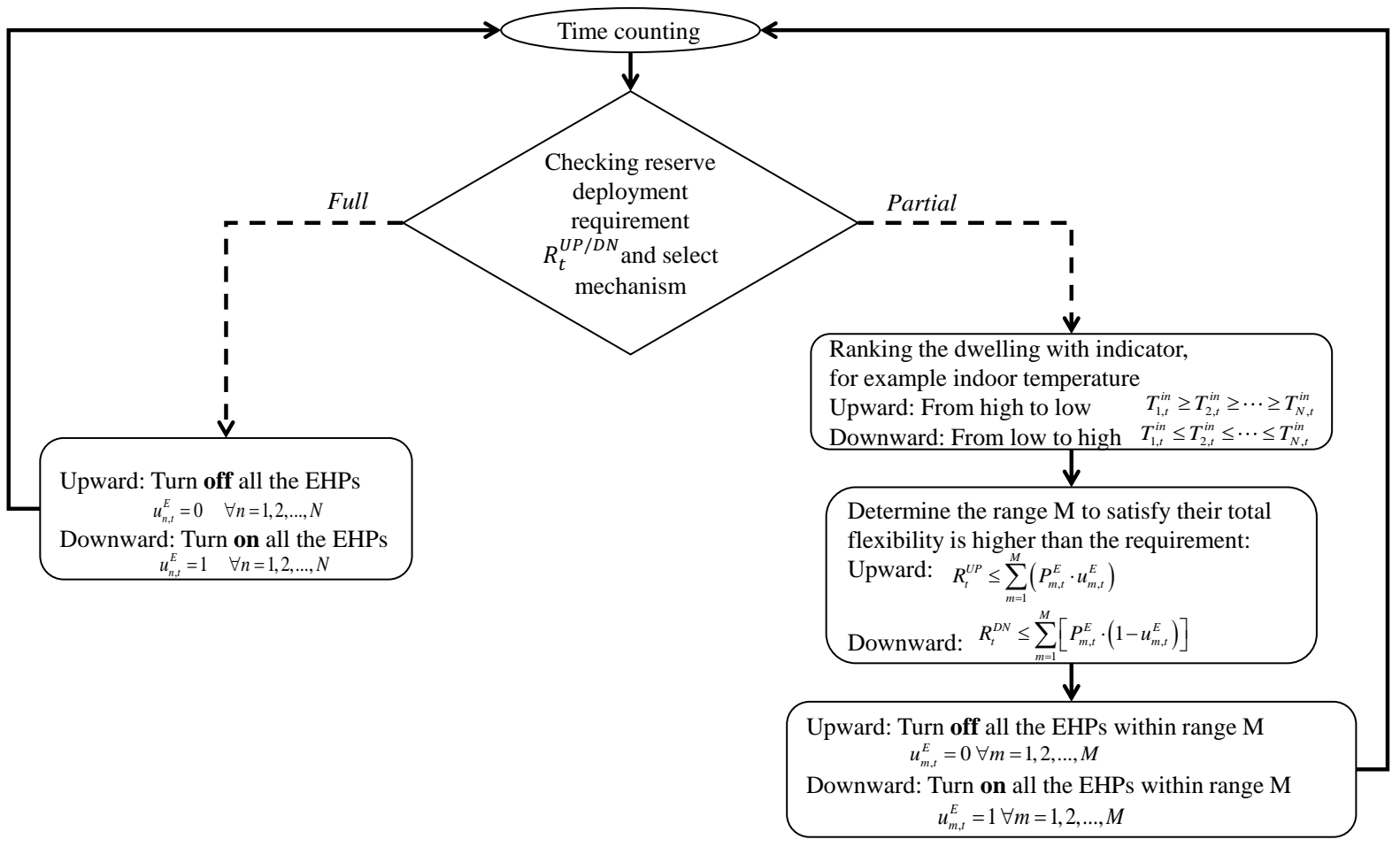

Figure 1. Flow chart of DR control mechanism

The average service capacity contributed by an EHP in the Partial/Full algorithms $\left(r_{t}^{U P / D N, P / F}\right)$ are determined in (16) and (17) respectively. The individual dwelling's flexibility is calculated with the cluster's upward or downward flexibility $\left(t f_{t}^{U P / D N}\right)$ within service windows $(w)$ and the number of dwellings in the clusters $(N)$. The service window periods are denoted as $W$.

$$
\begin{aligned}
& r_{t}^{U P / D N, P}=\min _{w \in W}\left\{\frac{t f_{w}^{U P / D N}}{N}\right\} \quad \forall t \in W \\
& r_{t}^{U P / D N, F}=\frac{t f_{t}^{U P / D N}}{N} \quad \forall t \in W
\end{aligned}
$$

\section{Model structure}

To assess flexibility from electric heating resources an understanding of the underlying physical constraints is important. The approach used here is to consider a 'bottom-up' model, starting from the capability and constraints of individual units. This model should be multi-energy in nature, to allow assessment of flexibility from energy vector shifting [24]. An example of such a model is that which is presented in [23]. This is a multi-energy, physically-based high resolution model that includes detailed building thermal modelling and can generate different energy vectors for households given a mix of technologies, partially built on the electricity-only model presented in [25]. Crucially for the assessment of flexibility potential, this model considers the thermal inertia of the building and heating system. A similar approach was followed in [26]. 
In this work an evolution of the residential energy consumption model presented in [23] is applied to simulate the performance of heating technologies (i.e., EHPs, gas boilers). As described by Figure 2, the constituent models are driven off key physical and user-related parameters which engender the ability to simulate a variety of cases, as desired. First of all, the input profiles are composed of parameters which can be randomly generated based on statistics. The "squares" in each module refer to the predefined parameters based on the model operator's choices on dwelling, emitter and season types, etc. An arrow between two sub-squares indicates the dependency of a parameter on another. An arrow between two modules in different steps indicates that the previous one is considered as an input for the analysis conducted in the following module. The space heating and domestic hot water (DHW) demands are considered as the two main heat consumptions. Following the end-user heat demand analysis, five distinct models are utilised: heat generation, cooking, electrical appliance, electric vehicle and photovoltaic. The "heat storage parameters", within the dashed line box, indicates that the storage is an optional configuration for the heating system of the dwelling. Correlations between the service demands are cultivated through the models' common basis in user activity profiles and environmental parameters. Further interactions between the models (such as between cooking and heat demand through cooking incidental heat gains) are also captured. Finally, the mapping between energy service demand profiles and dwelling energy profiles elucidates the multienergy aspect of the model. Note that the mapping of heat and cooking profiles to electricity and gas profiles depends on the heating and cooking technology selections.

The building and its heating system are modelled as electrical analogues with several nodes as explained in [23]. The value of the parameters, such as the thermal capacitance of indoor environment, the thermal resistance of walls, were initially generated with Design Builder and Build Desk $U$ software. Then, a calibration of parameters' value was carried out by using the survey data given in National Energy Efficiency Data framework until the difference of the dwelling's gas consumption in the simulation and the survey reference is less than $5 \%$. This can provide reassurance that, when considering aggregated results, the key uncertainties (which relate to building and heat emitter thermal characteristics) are not significant. The operation data of EHP is retrieved from [27] for modelling purpose, which considered the influence of source temperature and flow water temperature on input power and COP of the heat pump. In addition, the defrost cycle of heat pump in cold conditions is modelled based on the information provided in [28].

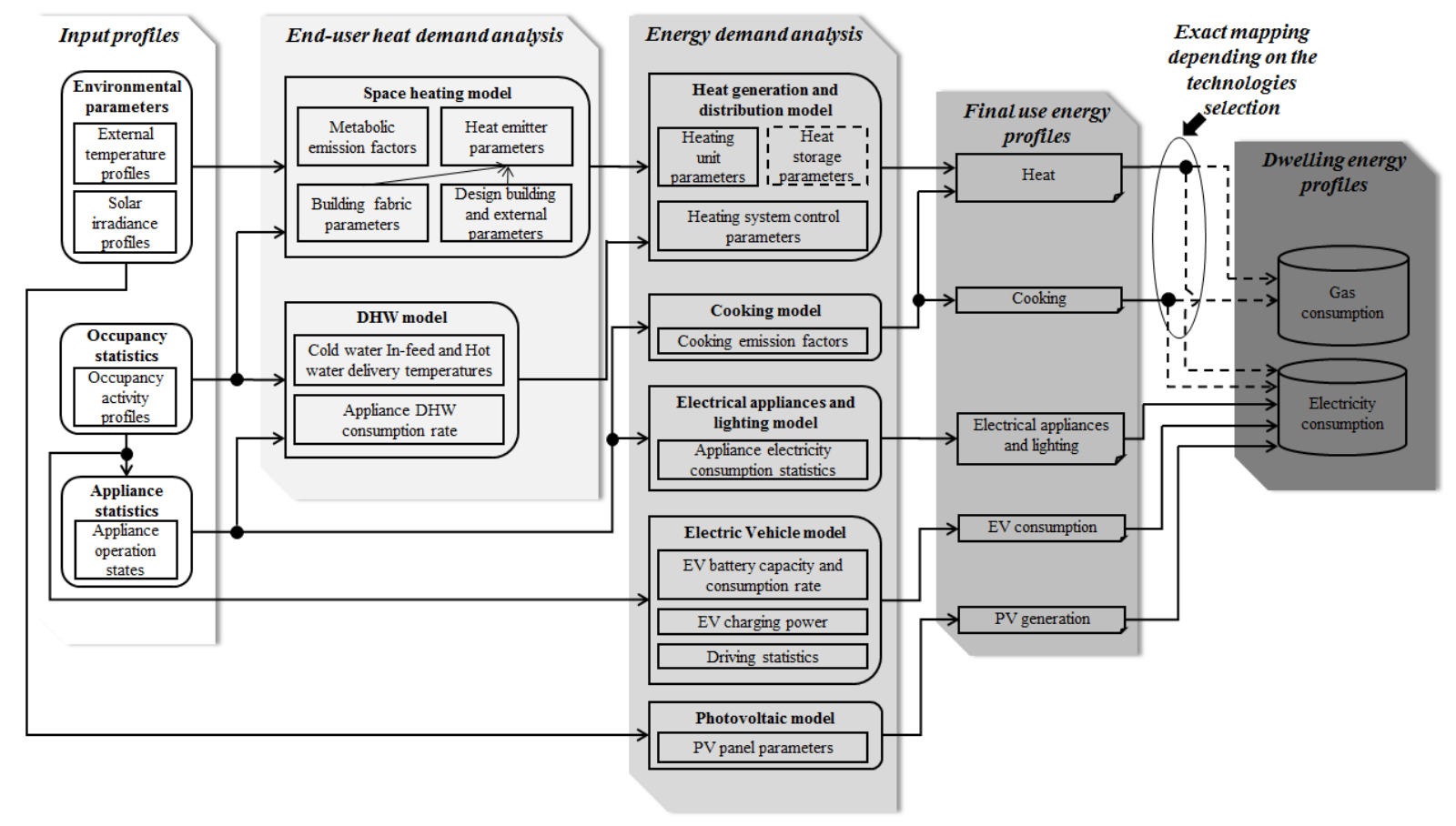




\section{Case studies and results}

For this case study both upward and downward flexibility services are designed based on National Grid (NG)'s short term operating reserve (STOR) product. As stated in the NG document [29], there are two relevant requirements for reserve service provision: First of all, the bidding capacity of reserve services needs to be more than $3 \mathrm{MW}$ generation or equivalent demand reduction from one tender. However NG also clarified that the capacity can be contributed from more than one site, which gives the possibility of the participation of an aggregator across different geographical regions or clusters. Secondly, the aggregator should be able to provide the bidding capacity during any STOR window listed in [30] with maximum two hours sustain periods. In order to examine the worst impact, the service sustain period is set to two hours following each window.

The configuration of dwellings and services in each scenario is shown in Figure 3. The scenarios are organised in such way to allow comparison in one dimension without influence from the variation of other factors. The examined four dimensions are explained as follows:

- $\quad$ Energy vector for heating

- Thermal inertia of dwelling and its heating system

- Deployed flexibility type

- Flexibility procurement mechanism

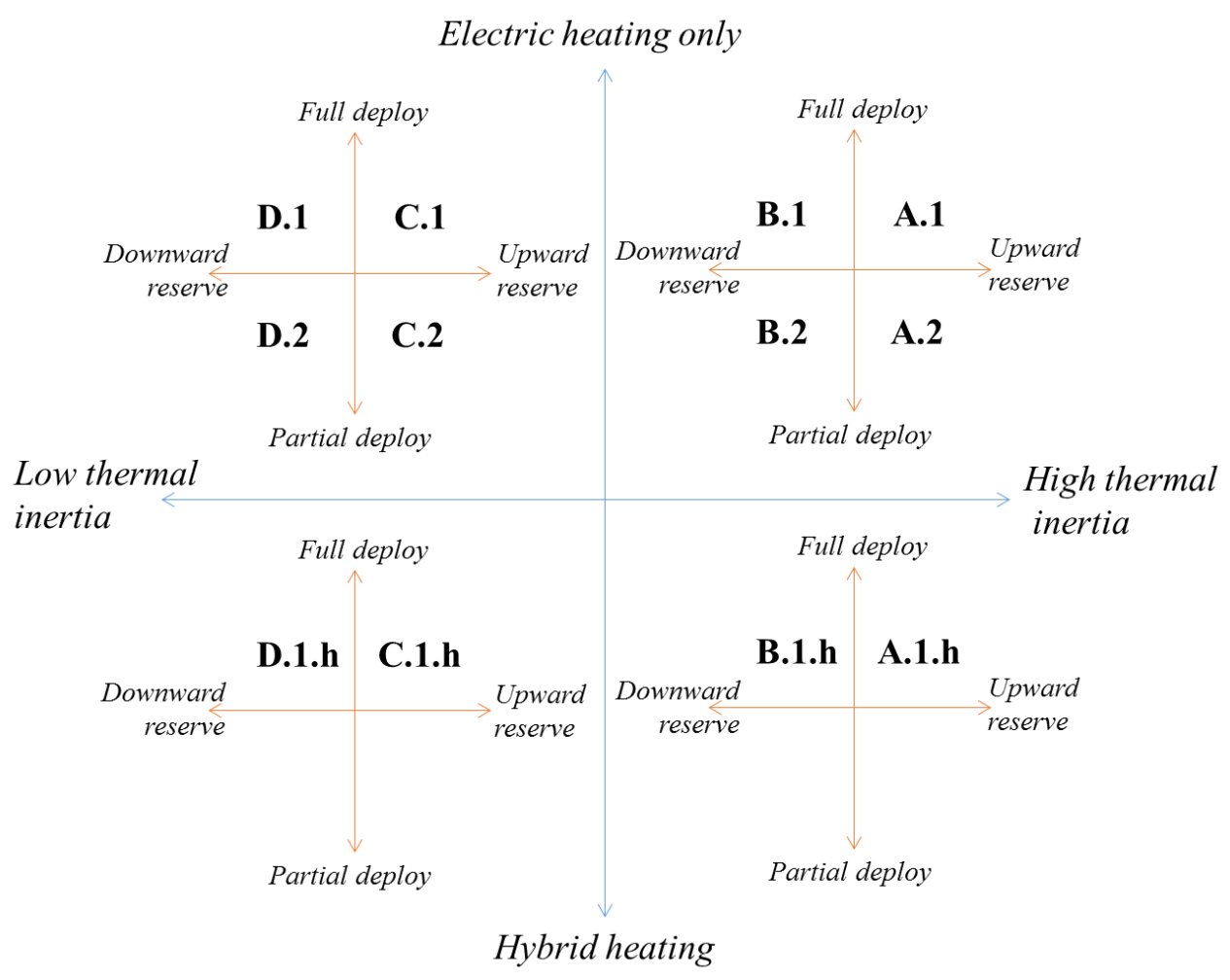

Figure 3. Configuration of scenarios compared from different perspectives.

Every dwelling is equipped with an air source heat pump (ASHP). The reason of choosing ASHP as the primary heating unit is that this type of EHP accounted $75 \%$ of heat pumps installation in the United Kingdom (UK) [31]. The power rating of ASHP is determined according to the thermal load of dwellings in the design conditions [23]. In the hybrid heating scenarios, a gas boiler acts as back up to the ASHP. Two types of dwelling and emitter combinations are investigated to represent buildings with different 
thermal inertia. The old detached dwelling and new flat represent the highest and lowest thermal demand dwellings in the database of the model [23]. Each flat has installed a radiator, while underfloor heating is used in the old detached house. A 300 litre buffer is installed with an underfloor heating system in order to reduce the cycling of the ASHP [22]. The indoor target temperature is set to $21^{\circ} \mathrm{C}$ during the occupant active period and $17^{\circ} \mathrm{C}$ for inactive period. A threshold control is applied to the heat provision for space heating buffer and DHW tank. The upper and lower temperature limits are 55 and $48^{\circ} \mathrm{C}$ respectively. The environmental parameters include the external temperature profile which is retrieved from historic data given in [32]. The weather pattern is for a typical UK winter weekday, which has a temperature range from $0.3^{\circ} \mathrm{C}$ to $5.4{ }^{\circ} \mathrm{C}$ as depicted in [32]. This temperature profile is uniformly applied to all dwellings, as we assume that the aggregator has procured the service from EHPs which are geographically close to each other in this case, also to address local network aspects. The solar irradiance data is generated by the model described in [33]. The number of dwellings in the cluster is set to 1000 , which is sufficiently large to give diversified results, as discussed in [23].

Both upward and downward reserve services with Full and Partial algorithms are examined in electric heating only scenarios, while only upward service with Full algorithm is analysed in the hybrid heating scenarios. This is because there is no shortage of heat supply in downward scenarios, which can be covered by the heat generation from gas boilers. In addition, the result of Full algorithm scenarios can indicate the upper limit of metrics in hybrid heating scenarios.

In the following content, the flexibility provision of the clusters is presented before the designed metrics are investigated for different scenarios displayed in Figure 3. External environment temperature profiles and an occupant's activity are the two factors which can result in different operation schedules of a dwelling's heating system and can consequently vary the value of different metrics. Therefore, the impact of these uncertainty factors on simulation results is also investigated in the following assessments. The external temperature is set as the random factor in the analysis of aggregator's metrics. This is because aggregators are interested in the potential disruption of DR participators' activity at different periods. Therefore, simulations are performed by setting DR activities at different service windows but with the same set of 1000 occupancy profiles in all scenarios to ensure a consistent comparison regarding the heat demand of occupants. Although the DR activity may involve more than 1000 dwellings in practice, the heating demands of occupants are welldiversified after reaching 1000 dwellings based on the results shown in [34]. For system and network operators, and retailers, the main challenges (such as capacity shortage and network congestions) generally happen at peak hours. Further, the peaks of heat demand and electricity are coincident, which means the comfort loss of DR participators can also be at the highest level during the evening peak period. Thus, the service window is fixed to 18:00 for the analyses of system/network operator, retailer and consumer related metrics. On the other hand, the impact of randomised occupant behaviour is considered in the assessment of operators, retailer and consumer related metrics. A Monte-Carlo simulation is performed to quantify the distribution of metrics value with different occupancy profiles. 500 rounds of simulation are undertaken for each scenario by randomly allocating occupancy profiles from a database composed of 5000 occupancy profiles.

\subsection{Flexibility provision}

The metrics related to aggregators are only analysed in electric heating only scenarios, while the results of the simulations in the hybrid heating scenarios (marked as '. $h$ ') are not presented here. This is because the auxiliary heater is only constantly activated when the heat loss rate of the dwelling is too high (i.e., in extreme cold days) or when the primary heating unit is not available (i.e., during DR events). However, the aggregator related metrics are evaluated based on the flexibility of the ASHPs 
operating in a normal condition before the service deployment. Therefore, the performance of ASHPs in both electric only and hybrid scenarios have almost the same performance ${ }^{3}$.

First of all, the upward flexibility of the ASHPs cluster and average indoor and external temperature profile are shown in Figure 4. It can be noticed in Figure 4 that the upward flexibility is substantially increased in 06:00-08:00 and 16:00-18:00, which is coincident with the period of occupants becoming active. In addition, the average indoor temperature is rising in these two periods as the heat supply increases. On the other hand, the flexibility decreases around midday. This is due to the heat demand reduction caused by a smaller temperature difference between indoor and external environments and the less active occupants in the dwelling on a weekday.

The average flexibility contributions from a dwelling in the clusters with the Full and Partial algorithms are depicted in Figure 5. For the Full algorithm, the service capacity is equal to the maximum available flexibility at each time step, which is marked as continuous line in. With regard to the Partial algorithm, the flexibility contribution is determined from the flexibility availability in all service windows and the service sustain period. For example, the upward service capacity with the Partial algorithm equals to the amount of available flexibility at 15:00 (Window 13:00 plus two hours sustain time), while the downward contract volume is determined by cluster's flexibility amount at 19:00. Moreover, the dwellings in scenarios A and B (left figure in Figure 5) have higher capacity contribution on both upward and downward services compared to scenarios $C$ and $D$ (right figure in Figure 5 ). This is due to the high thermal demand of the old detached dwelling compared with the new flat, which leads to a higher power rating of the EHP.
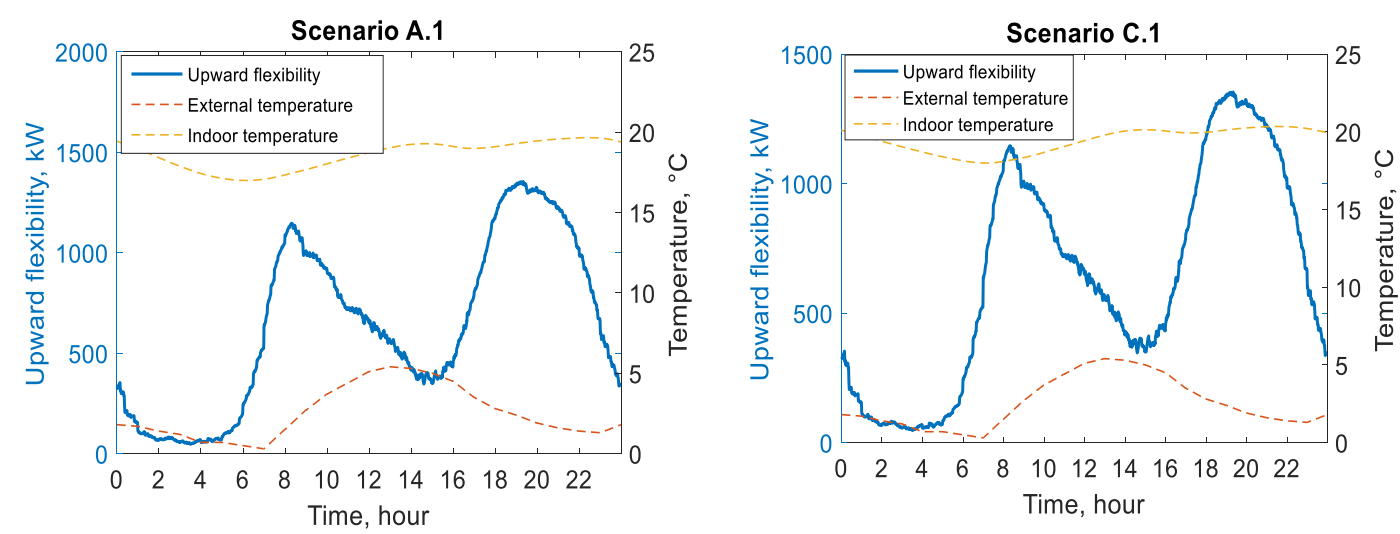

Figure 4. Maximum upward flexibility of EHPs in an old detached house (left) / a new flat (right), and the corresponding average indoor and outdoor temperature

\footnotetext{
${ }^{3}$ Although, the auxiliary heater may be shortly activated when the occupants are inactive for more than few hours and the emitter temperature is too low such that the emitter temperature can be brought back to a specific level quicker. But based on the analysis result this has minor impact on the ASHPs flexibility quantity and consequently ignored for this case study.
} 

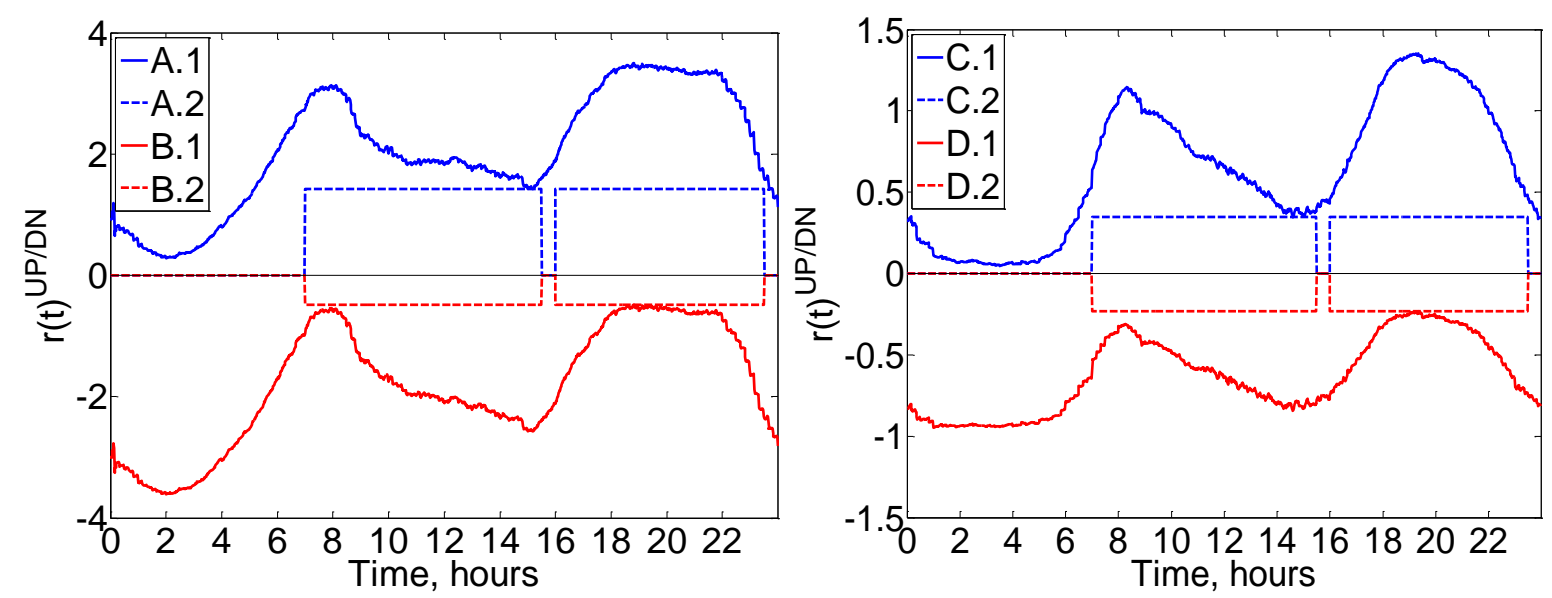

Figure 5. Average service provision capability from an EHP in an old detached house (left) / a new flat (right)

\subsection{Aggregator metrics}

The values of the three aggregator metrics (IDD, ADP and APC) are shown in Figure 6 and Figure 7, which separately consider the service provision from different clusters. For the IDP metric, the variation of its value in the scenarios with the Partial algorithm (A.2, B.2, C.2 and D.2) is more volatile in the morning services windows compared to the metric value in afternoon and evening ones, as shown in Figure 6 and Figure 7. This phenomenon indicates that more customers are expected to be interrupted for services provision with the Partial algorithm in the morning. The reason for the higher customer interruption percentage is that the Partial algorithm uses the indoor temperature level to decide the switching ranking of ASHPs in the service provision process. In the morning, the state of dwellings (i.e., indoor temperature) are 'synchronised' after an overnight inactive period. This causes the ranking list in the algorithm to be frequently adjusted after the disruption of heating activity. Consequently, the range of impacted customers is widened. On the other hand, the IDP value in the upward service scenarios with the Full algorithm (A.1 and C.1) identifies the percentage of customers with heat demand, while the value of the metric in the downward service scenarios (B.1 and D.1) indicates the percentage of customers whose heating system is operating intermittently. As seen in Figure 6 and Figure 7, the variation of the IDP metric across the day shows a contrary trend in upward and downward scenarios. For example, the IDP metric in A.1 and C.1 scenarios reaches its peak values at around 07:00 and 18:00, while the peak of IDP metric in B.1 and D.1 scenarios happens at 13:00. This finding indicates the highest and lowest heat demand periods in the service windows.

The value of ADD metric is also shown in Figure 6 and Figure 7. Based on the ADD metric value in the scenarios with the Full algorithm (A.1, B.1, C.1 and D.1), it can be interpreted that the average operating duration of the ASHP at each window is in the range of 60 to 90 minutes. The maximum ASHP operation duration happens at 07:00 and 18:00, while the minimum duration occurs at 13:00. These time windows are also coincident with the "peak and valley" times of customers heat demand.

The value of the APC metric is shown in Figure 6 and Figure 7 as well. The APC metric shows a constant performance in scenarios $C$ and $D$, while scenario $B$ has a higher APC metric value than the result in scenario $A$. This is due to the additional buffer component in the old detached house case, which gives the possibility of reaching higher water temperature in the heating system for downward reserve case and consequently higher input power of EHP to enable same level of heat with low coefficient of performance (COP) [35]. 

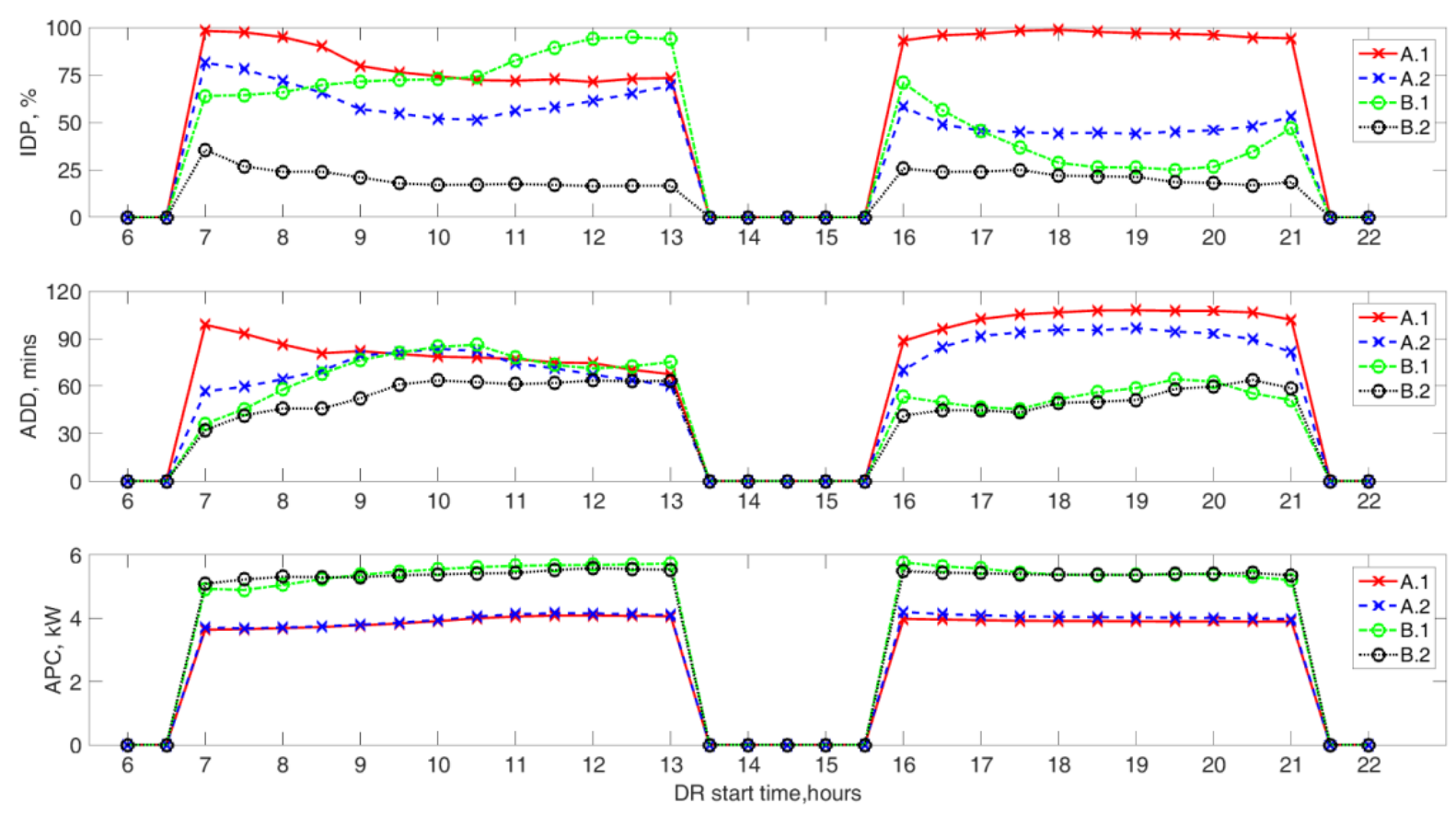

Figure 6. Aggregator metrics performance of a 1000 old detached dwelling cluster at service windows
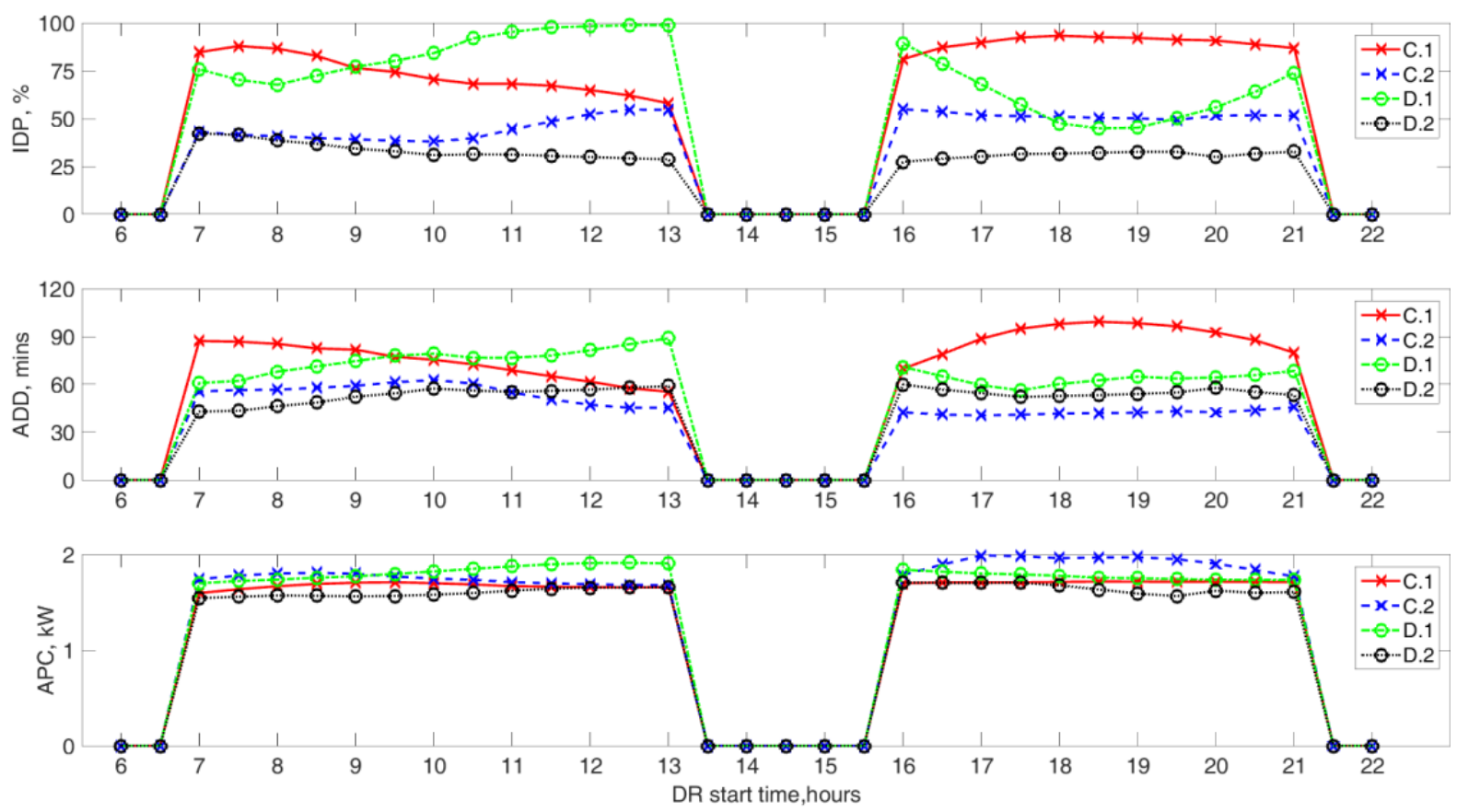

Figure 7. Aggregator metrics performance of a 1000 new flats cluster at STOR windows

\subsection{Operators and retailer metrics}

The metrics designed for SO, TNO, DNO and retailer are analysed with the simulation of different scenarios. Firstly the CMR metric is analysed to show how much flexibility is not exploited by an ancillary service, especially when the service capacity is required to be the same across the window. Subsequently, other metrics are considered. Because the operators and retailer are more concerned of the worst impact caused by services provision (such as network congestion and market bidding 
imbalance), only the scenarios with the Full algorithm are investigated in this section, which deployed all available flexibility in the cluster.

\subsubsection{CMR metric}

By comparing the deployable flexibility in the two algorithms shown in Figure 5 the values of CMR metric can be calculated (4). It can be seen in Figure 8 that the maximum value of CMR metric is only $47.2 \%$ for upward reserve service provision from the old detached dwellings cluster. This means that only $47.2 \%$ of available upward flexibility of EHPs installed in a detached house is being used for the corresponding grid service provision. This can lead to the conclusion that the maximum volume contracted by an aggregator for a STOR-type service is substantially smaller than the sum of the clusters' available flexibility.

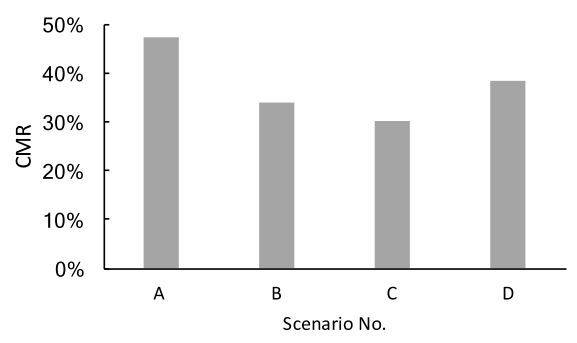

Figure 8. CMR metrics of different dwelling clusters with upward/downward services provision

\subsubsection{Electric heating only cluster}

First of all, the performance of the ASHP only clusters is analysed. The PPR, EPR and TECR values are plotted in normal distribution and shown in Figure 9. With regard to the PPR metric, when the value of PPR metric is negative it indicates a reduction of the peak power of the cluster with the DR activation, and vice versa for the positive value. In Figure 9, only the scenario A.1 has a negative median value ($5 \%$ ), while the maximum PPR metric median value is $35 \%$ in the scenario D.1. For the upward service scenarios (A.1 and C.1), it can be noticed that the change of peak power is different for cases A.1 and C.1, with reduction in the scenario A.1 and increase in the scenario C.1. This is due to the thermal characteristics of the dwellings and their corresponding heating system in the cluster.
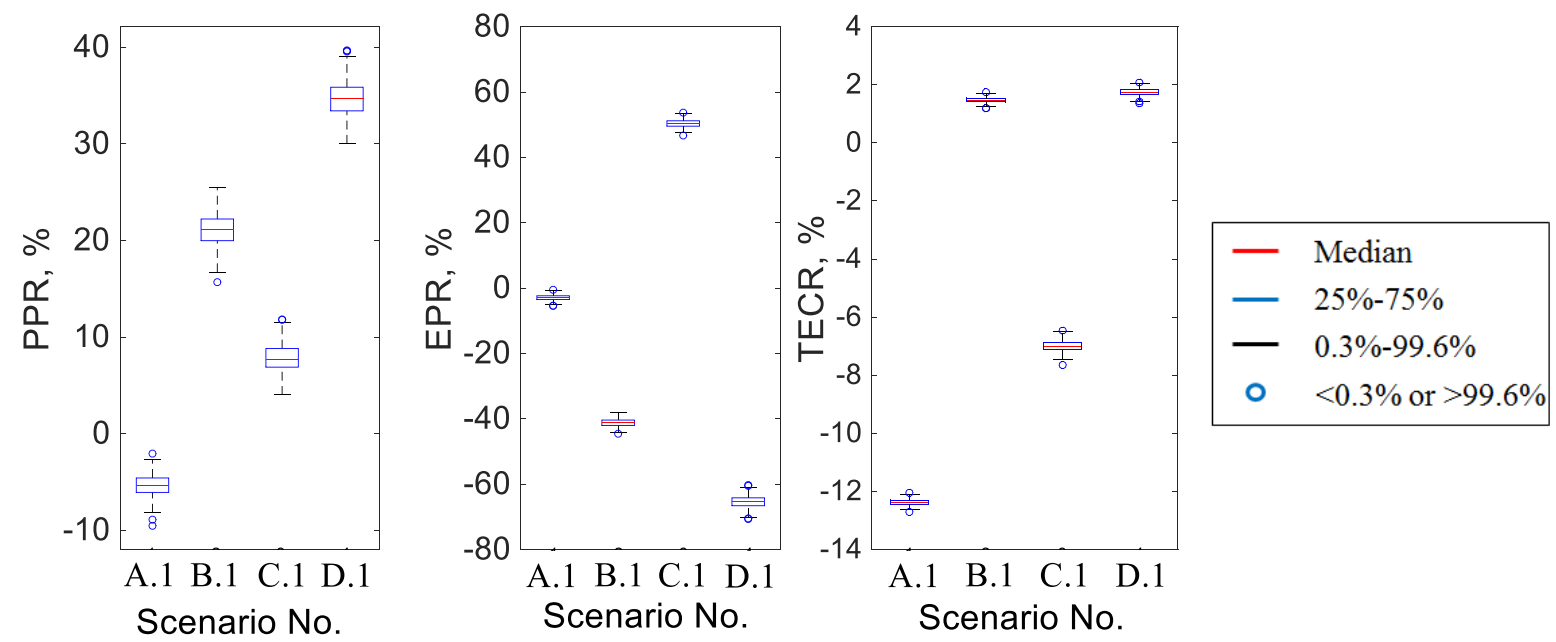

Figure 9. Distribution of operator and retailer's metrics value, in ASHP only cluster, with Full algorithm service provision between 18:00 and 20:00

In order to facilitate the understanding of different payback phenomenon, the synchronisation rate, average input power of ASHP and, in the upward service scenarios (A.1 and C.1), the average indoor temperature are shown Figure 10. The value of synchronisation rate shows the percentage of 
activated ASHP in the cluster, while the input power for individual ASHP is determined with the external air temperature and return water temperature in the heating system, as explained in [23]. It has been detailed in [23], the building model uses electrical analogues to capture the thermal characteristics of both dwellings and installed emitters. Therefore, the delay of heat transfer from heating generation to emitters has been properly represented, which produces a realistic variation of indoor temperature following a DR event. The aggregated input power of the cluster is determined using the average input power and the activated ASHP in the cluster. A negative PPR value in scenario A.1 is caused by two factors: 1) Small synchronisation rate increase; 2) Slow ASHP input power recovery. The underfloor heating system is designed for continuous operation and the synchronisation rate is already high (around $85 \%$ ) in the BaU case, therefore the increment of synchronisation rate following an upward DR event is relatively small. Meanwhile, the temperature of the return water in the heating system is substantially reduced during a DR period. Also, the temperature recovery of the heating system takes longer in a high thermal inertia system (such as old detached house equipped with underfloor heating), which subsequently leads to a slower increase of ASHP input power. Thus, the payback power peak is reduced in high thermal inertia scenario A.1. On the other hand, a higher power peak is detected in the low thermal inertia scenario C.1, while the cluster's daily peak power can increase $12.5 \%$, which is caused by a larger synchronisation rate increase and a faster input power recovery as seen in Figure 10 . More importantly, two distinct phenomena can be observed by comparing the indoor temperature of scenario A.1 and C.1. Comparing the indoor temperature results in Figure 10 show that the temperature decreases slower during the service provision period and slower recovery after service provision completed in scenario C.1 compared to scenario A.1. This is due to the different levels of thermal inertia of dwellings. In scenario A.1, the high thermal inertia of old detached house has helped to maintain the indoor temperature without heat supply from ASHPs. However, due to its high thermal inertia, the indoor temperature of old detached house is also harder to be increased. Unlike the old detached house, the low inertia new flat means that the indoor temperature is more responsive to heat supply adjustment.

For the downward services, the PPR metric value can reach to $25.5 \%$ and $40 \%$ in scenarios B.1 and D.1 respectively, as seen in Figure 9 . The downward service can be called by retailers to reduce its market bidding imbalance, or by system operators to absorb excess renewable generation. However, the system and network operators also need to recognise the potential risk posed by downward services on network congestions due to high power paybacks. The impact of randomness of occupancy activities on the PPR metric value is shown in Figure 9. The standard deviation of the PPR value distribution in the upward service provision scenarios (A.1 and C.1) are around $0.7 \%$ to $1.2 \%$, while the downward services scenarios (B.1 and D.1) have 1.5\% standard deviation. 

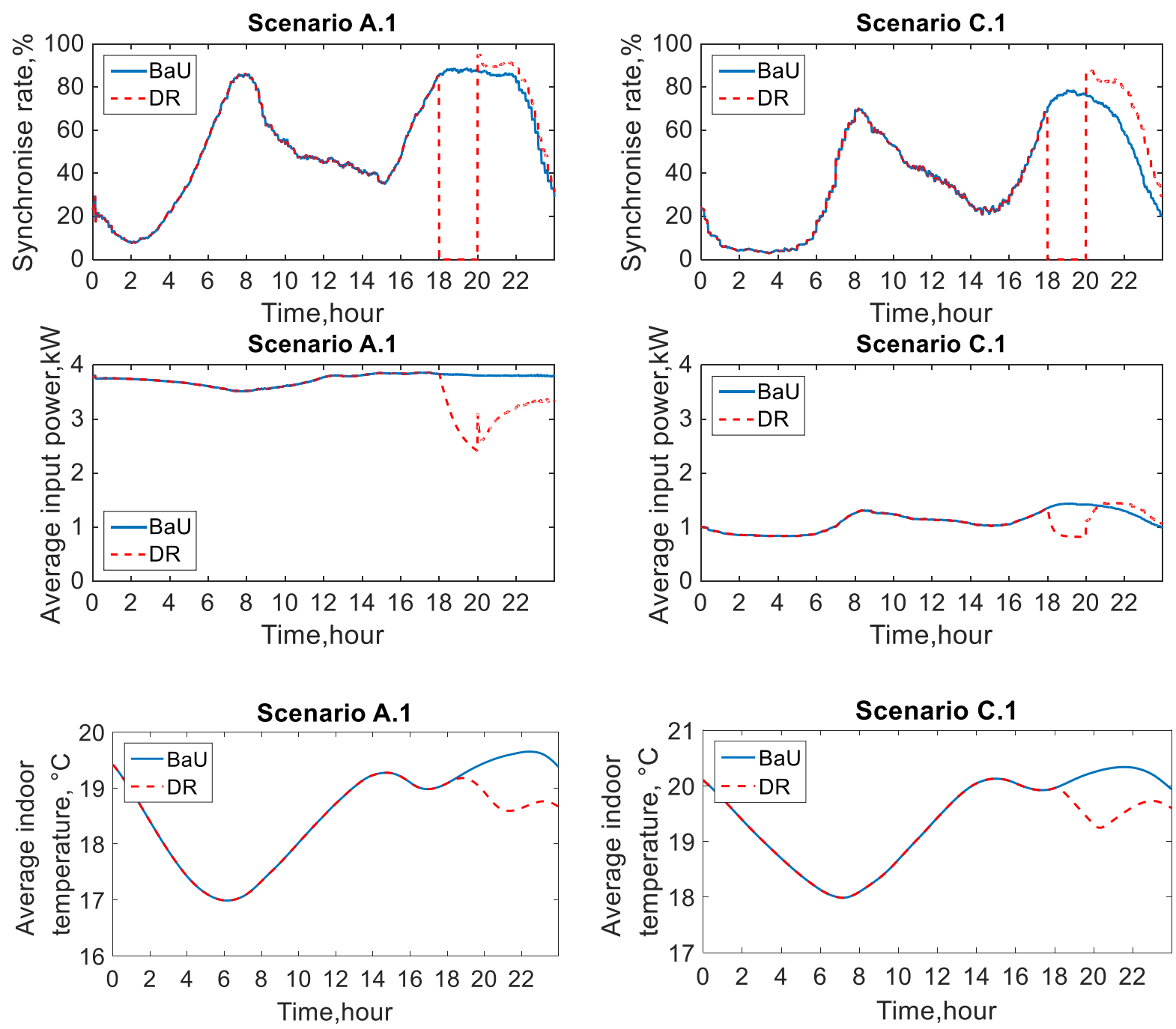

Figure 10. Synchronisation rate and average ASHP input power for Scenario A.1 (left) and Scenario C.1 (right) in normal operation or with Full upward reserve between 18:00 and 20:00

Secondly, for the EPR metric, a positive value implies an increase of electricity consumption after a DR event and vice versa for the scenarios with a negative EPR. In Figure 9, the scenario A.1 has a negative EPR with $-2.2 \%$ median value. This is because of the slower recovery of ASHP input power in the high thermal inertia dwellings as discussed above, so there is no payback effect for the upward service provided by the old detached houses cluster. In the scenario with new flats, the EPR median value in the scenario C.1 is $50 \%$, which indicates that half of the electricity curtailment in DR event is "recovered" in the following hours after the service provision. The EPR values in the downward service scenarios (B.1 and D.1) can reach to $-45 \%$ and $-70 \%$ respectively. The reason for the higher reduction in electricity consumption following the downward service event (corresponding to -70\% EPR values) in the scenario D.1 is that the new flat has a better insulation level and consequently lower thermal loss during the service period. In the downward service provision process, the indoor temperature increases due to the excess heat generation and the preservation of stored heat in the well-insulated dwelling, which leads to the reduction of heat requirement in the following hours. The standard deviation of EPR metric values is in the range of $0.5 \%$ to $1 \%$ which is slightly smaller than the standard deviation shown in the PPR metric values distribution.

The TECR metric is analysed in different scenarios. It can be seen in Figure 9 that the maximum magnitude of TECR metric in scenario A.1 and C.1 are $-13 \%$ and $-8 \%$ respectively. The higher reduction of total energy consumption in scenario $A .1$ is caused by the no payback effect in scenario A.1 while 
the EPR in scenario C.1 is $50 \%$. It also needs to be emphasised that this metric informed the potential revenue loss of retailers due to the DR service carried by aggregators, as retailer's turnover mainly depends on the amount of customer's energy consumption. The TECR metric in downward service scenarios has a smaller magnitude, with median value less than $2 \%$. This is because the downward flexibility is limited between 18:00 and 20:00, which leads to a smaller total energy consumption change in DR event compared with BaU case.

At the network planning stage, one of the most important indicators for network design is the coincidence factor. The coincidence factor can measure the diversification of electricity consumption profiles of individual households in the same network, which determines the rating requirement of lines and transformers in the network. The simulation has been carried out one thousand times for each number of houses. Only the maximum coincidence factor of the results in these one thousand rounds is preserved which should reflect the worst situation of the network congestion. The base load profile from residential appliances is also included to produce the full electricity consumption profile of dwellings. Figure 11 shows the variation of the coincidence factor against the number of dwellings in four scenarios (A.1, B.1, C.1 and D.1). The load profiles in the BaU scenario represent the electricity consumption of the corresponding dwellings (old detached houses/new flats) without DR application. Firstly, it can be noticed that the coincidence factor in the old detached dwellings BaU scenario is 0.525 at one thousand dwellings aggregated level. This is higher than the coincidence factor at same aggregated level in new flats scenario, which equals to 0.333 . The coincidence factor is higher in the old detached dwellings with underfloor heating scenario, because the ASHPs are operating more frequently in this cluster, which results in a higher synchronisation rate in the left subfigure of Figure 10. In addition, the coincidence factors of the scenarios with/without DR application overlap with each other at low aggregated level (e.g. 10-15 dwellings). This means the DR application would not lead to a substantial increase in coincidence factor for a small number of buildings, although the power increase due to payback effect needs to be considered in the network rating determination process. Furthermore, the upward service provision is also not leading to a substantial variation on the coincidence factor. For example, the coincidence factor is only increased by 0.003 at the saturated level in scenario C. 1 compared to its BaU scenario, while the coincidence factor is reduced by 0.015 in the scenario A.1. On the other hand, the provision of downward service at peak hour can lead to a substantial increase on the coincidence factor. As shown in Figure 11, the coincidence factor has increased to 0.613 and 0.399 in scenarios B.1 and D.1 respectively. Therefore, the potential network congestion issue needs to be considered during the downward DR activation periods.
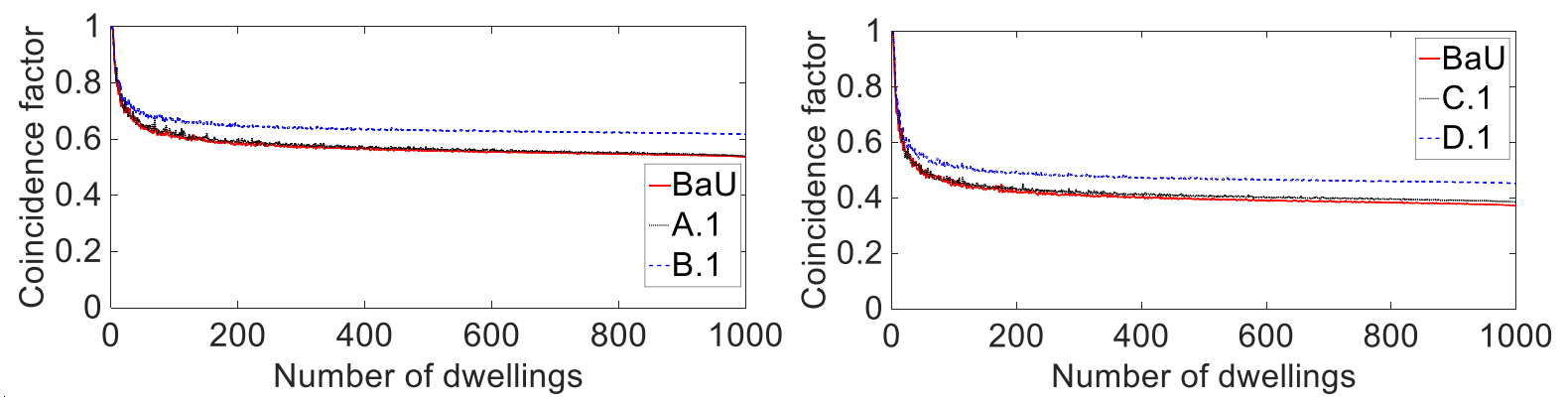

Figure 11. Coincidence factor of the BaU and reserve provision between 18:00 and 20:00, old detached houses with underfloor heating (left) and new flats with radiator (right)

\subsubsection{Hybrid heating cluster}

In addition, the performance of a hybrid heating system is also simulated and the corresponding metrics value is depicted in Figure 12. The first metric introduced is the NETR, which is only relevant for the hybrid heating scenario as it concerns electricity and gas consumption. Its value in upward metrics is around $195 \%$. This means gas consumption in upward reserve scenario is around twice of 
the electricity consumption in the BaU case during reserve provision period. With regard to downward reserve scenarios, it can be noticed that even with additional heat supply due to ASHP ramping up, Scenario B.1.h has almost no gas consumption reduction while the reduction of gas in Scenario D.1.h is also only around $70 \%$ of the quantity of additional electricity consumption. The reason for the low gas saving in downward reserve scenario is that the idle ASHPs, which is used to provide downward reserve, may belong to the dwellings without heat demand after reserve provision period. However, the gas reduction is more visible $(70 \%)$ in the radiator scenario (Scenario D.1.h) compared with the underfloor heating scenario (Scenario B.1.h). This is because the emitter temperature and the indoor temperature are maintained to the required level in the dwelling with high thermal inertia heating system, while the gas boiler is occasionally activated in the radiator scenario due to the low thermal inertia in both flat building fabric and the emitter. The PPR metrics in hybrid heating scenarios are also shown in Figure 12. It can be noticed that there is no difference on the distribution of PPR values in the downward scenarios (Scenarios B.1.h and D.1.h) compared to the results of the hybrid heating cluster in Figure 12 and the results of the EHP only cluster in Figure 9. This is because no extra heat provision is required for the downward service scenarios and there is consequently no impact or benefit of the gas boiler's existence. For the upward scenarios, the median value of the PPR metric are increased from $-5 \%$ to $0 \%$ and from $7.5 \%$ to $15 \%$ in Scenario A.1.h and C.1.h respectively. This is because the indoor temperature and the temperature of buffer/radiator are maintained at a higher level compared with the EHP only scenarios, such that the initial input power of the ASHP is higher in the hybrid heating scenarios and lead to a higher payback. For the EPR metric, the distribution of the value in the downward scenarios is similar to the EHP only scenarios and its median values are $45 \%$ and $65 \%$ for Scenario B.1.h and D.1.h respectively. However, the energy payback is increased in Scenario A.1.h because the gas boiler has maintained the buffer temperature, which results in a higher initial ASHP input power as explained above. For Scenario C.1.h, the median value of energy payback metric is reduced from $50 \%$ to $18 \%$ in comparison with the result of the electric heating only scenario (Scenario C.1), as the heat supply of the gas boiler during reserve provision period has reduced the heat requirement in the post reserve period.
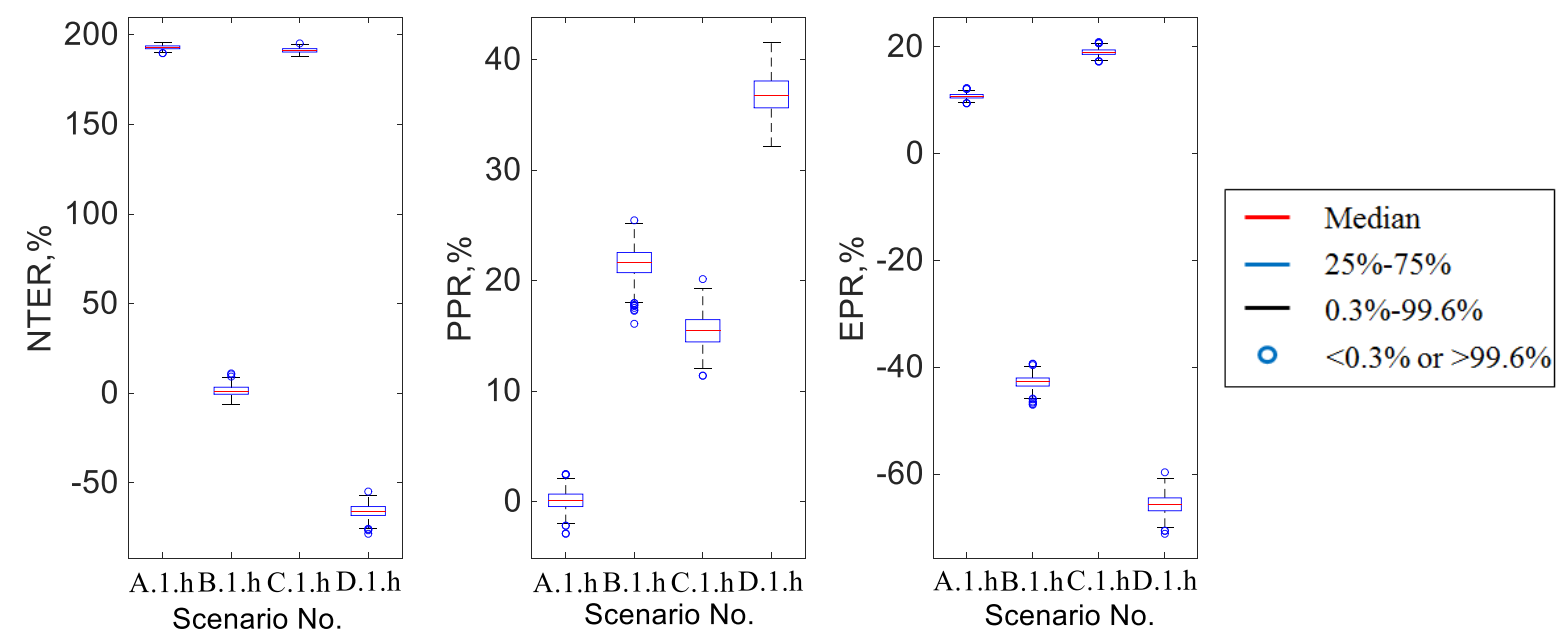

Figure 12. Distribution of NETR, PPR and EPR metric values, hybrid system, Full algorithm between 18:00 and 20:00

\subsection{Consumer metric}

Figure 13 depicts the probability distribution of a household's comfort variations in different reserve provision events. For upward reserve provision scenarios (Scenarios A.1 and C.1), the maximum comfort loss can reach to $-1.1^{\circ} \mathrm{C}$. Although, the probability of having same level of the comfort loss is lower in a high thermal inertia dwelling (Scenario A.1) compared to a low thermal inertia one (Scenario C.1). For example, there is $80 \%$ probability of having a comfort loss in a detached dwelling compared with $90 \%$ in the new flat scenario, as shown in Figure 13. It shows that the combination of high thermal 
inertia dwelling and heating system can help to reduce the impact of heat supply shortage on occupant's comfort. In addition, there is even a small possibility of comfort gain in the upward reserve provision events, which are $1 \%$ and $2 \%$ for Scenarios A.1 and C.1 respectively. This heat gain phenomenon only happened to a dwelling with small heat demand curtailment when this load curtailment accidently induced a new operation cycle of the heating system, which subsequently leads to a slightly better comfort level. For the downward reserve scenarios (Scenarios B.1 and D.1), the probability of having a comfort loss is smaller than the upward reserve scenarios. Moreover, a high thermal inertia dwelling has no comfort loss possibility and maximum probability of a comfort gain is $20 \%$. This is due to the lack of spare capacity during peak periods. As depicted in Figure 10, the synchronisation rate of the ASHPs reached $85 \%$ between 18:00 and 20:00 in Scenario B.1 and therefore less idle ASHPs are activated and supply unnecessary heat to dwellings. However, in the low thermal inertia dwelling scenario, the probability of comfort loss and gain reaches to $10 \%$ and $30 \%$ respectively, and the comfort gain can be as high as $1.5^{\circ} \mathrm{C}$. This means the downward reserve provision has achieved a better occupant's comfort in the following hours and there is even a possibility with overheating by $0.5^{\circ} \mathrm{C}$ as shown in Figure 13. As mentioned above, the comfort loss of a dwelling with a hybrid heating system is also analysed and shown in Figure 14. In upward reserve provision scenarios (A.1.h and C.1.h), the maximum comfort loss is tremendously decreased from $-1.1{ }^{\circ} \mathrm{C}$ to $-0.4{ }^{\circ} \mathrm{C}$ and $0.3^{\circ} \mathrm{C}$ respectively. Furthermore, the probability that a dwelling has a comfort loss has reduced to $20 \%$ and $10 \%$ in Scenarios A.1.h and C.1.h. With regard to downward reserve provision, by comparing Scenarios B and D in Figure 13 and Figure 14, it can be concluded that the additional gas boiler does not have a significant impact on the probability distribution of occupant comfort loss.
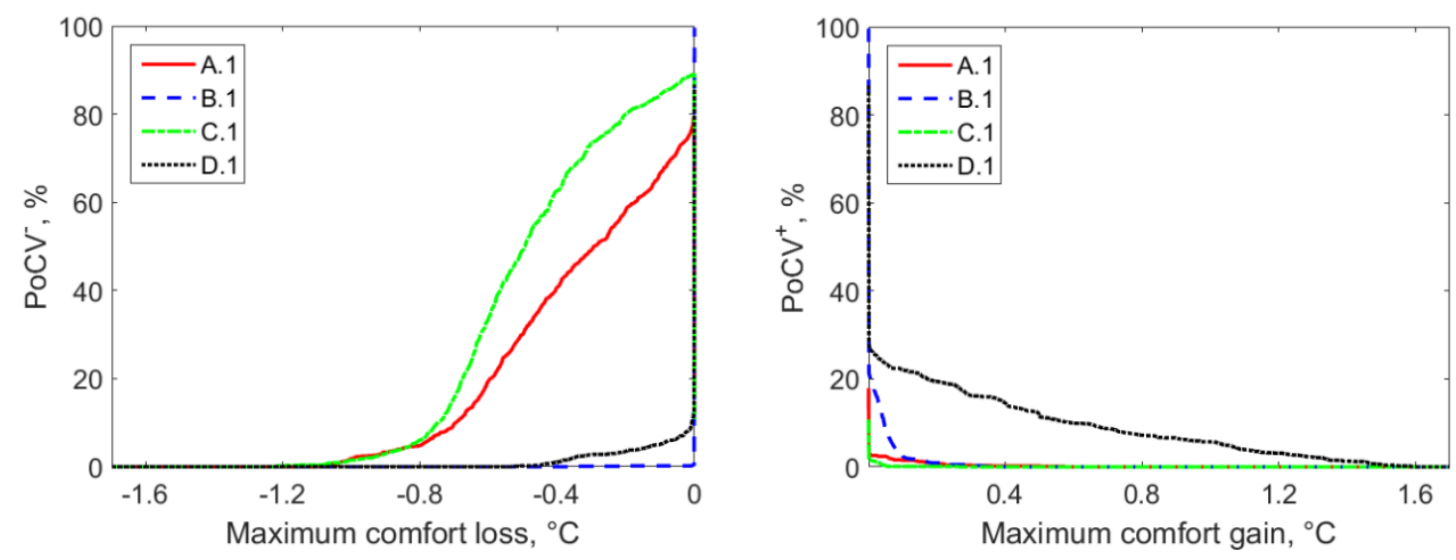

Figure 13. Probability distribution of maximum occupant's comfort changes, EHP only system, Full reserve between 18:00 and 20:00
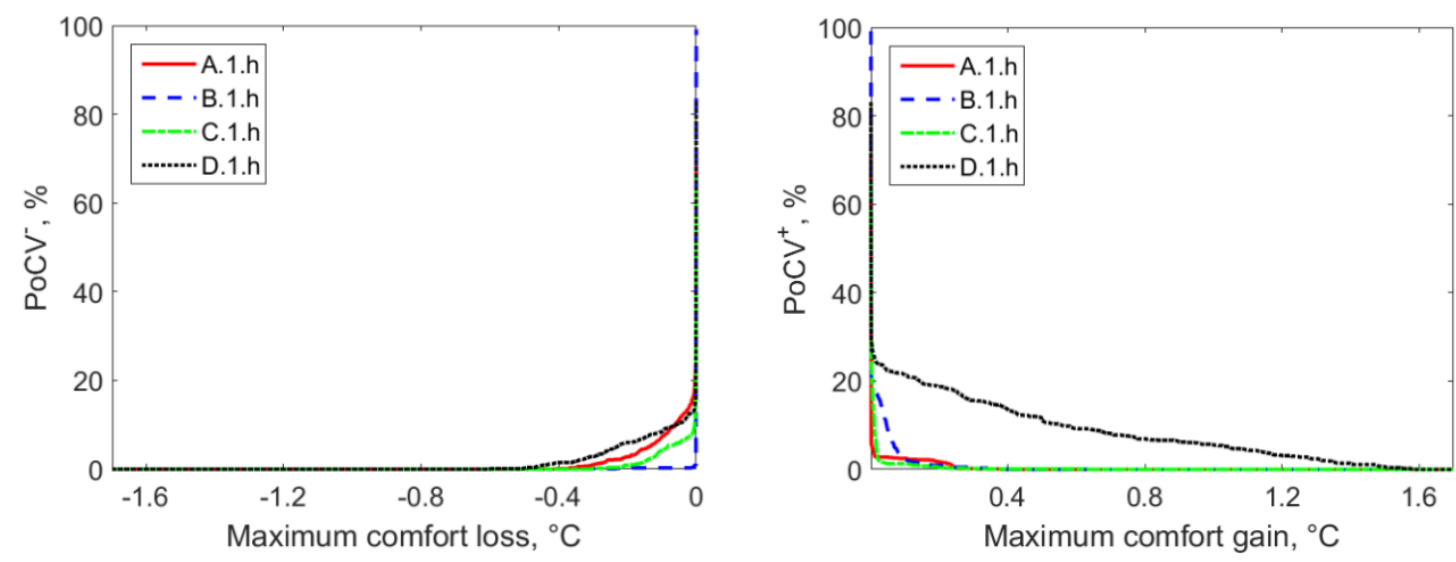

Figure 14. Probability distribution of maximum occupant's comfort changes, hybrid system, Full algorithm between 18:00 and 20:00 


\section{Conclusion}

This paper has proposed a unified framework to assess the building-to-grid flexibility embedded in future residential EHP clusters and the corresponding potential to provide different DR services to different stakeholders. As a key contribution, several metrics have been proposed to comprehensively assess the performance of EHP clusters in providing services from the perspective of the different stakeholders involved. These metrics are designed to concisely communicate how DR will impact each stakeholder. For the system and network operators the PPR and coincidence factor metrics define how peak power demand and load diversity will be affected. Further, the CMR metric describes how much flexibility is not accessed due to the requirement to provide the same amount of flexibility across the service window. For retailers, there are metrics that define the changes in energy consumption. These include changes in electricity consumption as a function of the amount of DR delivered, the absolute changes and the amount switched to the alternative energy vector, gas. For aggregators, metrics provide means to remunerate consumers for the provision of DR. For consumers the PoCV metric defines the loss of comfort due to DR provision, which is crucial to understanding the value of DR provision to the consumer. Additionally, two service deployment methods, which employ different trade-offs between efficacy and privacy, have also been introduced.

In order to demonstrate the metrics and algorithms introduced, a physically based residential energy consumption model was adopted. Case study applications in the UK context were run and analysed, which demonstrated the utility of the proposed framework. Case studies show how the maximum utilised percentage of aggregated EHP flexibility for the STOR service is less than $50 \%$ in winter, as the maximum value of the CMR metric is $47.2 \%$ in scenario $A$. This is due to the variability of the aggregated load of EHP clusters and STOR service requirement. This is an important result as it demonstrates how a restrictive ancillary service product definition can result in available flexibility being unused. We also found that a cluster's upward reserve provision activity does not necessarily result in positive energy and power paybacks for ASHP installed in dwellings with high thermal inertia, such as the old detached dwelling with underfloor heating system and buffer shown above. By providing downward reserve, the cluster can also substantially reduce its electrical demand in the following hours, which demonstrated the effectiveness of using EHP cluster to absorb excess renewable generation. This information of demand variation can be vital to SOs and NOs whose are responsible for balancing the system and avoiding network congestion. In this case, the SOs and NOs can be prepared for the activation of EHP DR applications. The probability distribution of the occupant's comfort loss shows that the effect of reserve provision is on the occupancy state of the dwelling before reserve deployment period. In the case studies examined above, the maximum comfort loss can reach to $1.1^{\circ} \mathrm{C}$. Last but not least, the usage of additional gas boiler can switch the heat supply from electricity to gas. This would reduce both payback phenomenon on electrical system and comfort loss.

Based on the results shown above, several recommendations may be made to improve the operation and planning of energy systems with flexible heat pump clusters:

- Considering the flexibility potential of the domestic sector, regulation should be updated to allow maximum deployment this flexibility in ancillary services markets.

- The clusters can be used to relieve the burden on conventional generators to provide ancillary services, so that generators can operate at a more efficient point with less cycling times and consequently bring economic and environmental benefits to the power system. Based on increasing interactions between different energy vectors, coordination of the multi-energy system should be carried out to minimise overall cost across different energy systems.

- There are side effects of DR provided by flexible electric heating, such as payback and comfort loss, which also vary with the characteristic of dwellings. Optimisation algorithm could be designed based on the models developed that could strike a fine balance between maximising 
flexibility deployment and minimising the corresponding negative impact. This will be a focus of future work.

\section{Acknowledgement}

This work was developed with the contribution of the UK EPSRC MY-STORE project (ref. no EP/N001974/1).

\section{Reference}

[1] Zhang L, Capuder T, Mancarella P. Unified Unit Commitment Formulation and Fast MultiService LP Model for Flexibility Evaluation in Sustainable Power Systems. IEEE Trans Sustain Energy 2016;7:658-71. doi:10.1109/TSTE.2015.2497411.

[2] Department for Business Energy and Industrial Strategy. Energy Consumption in the UK. London: 2016.

[3] Sharma S, Huang S-H, Sarma N. System Inertial Frequency Response estimation and impact of renewable resources in ERCOT interconnection. 2011 IEEE Power Energy Soc. Gen. Meet., IEEE; 2011, p. 1-6. doi:10.1109/PES.2011.6038993.

[4] Qazi HW, Flynn D. Analysing the impact of large-scale decentralised demand side response on frequency stability. Int J Electr Power Energy Syst 2016;80:1-9. doi:10.1016/j.ijepes.2015.11.115.

[5] Lu N, Zhang Y. Design considerations of a centralized load controller using thermostatically controlled appliances for continuous regulation reserves. IEEE Trans Smart Grid 2013;4:91421. doi:10.1109/TSG.2012.2222944.

[6] Nistor S, Wu J, Sooriyabandara M, Ekanayake J. Capability of smart appliances to provide reserve services. Appl Energy 2015;138:590-7. doi:10.1016/j.apenergy.2014.09.011.

[7] Liu W, Wu Q, Wen F, Ostergaard J. Day-Ahead Congestion Management in Distribution Systems Through Household Demand Response and Distribution Congestion Prices. IEEE Trans Smart Grid 2014;5:2739-47. doi:10.1109/TSG.2014.2336093.

[8] Hejeejo R, Qiu J. Probabilistic transmission expansion planning considering distributed generation and demand response programs. IET Renew Power Gener 2017;11:650-8. doi:10.1049/iet-rpg.2016.0725.

[9] Dagoumas AS, Polemis ML. An integrated model for assessing electricity retailer's profitability with demand response. Appl Energy 2017;198:49-64. doi:10.1016/j.apenergy.2017.04.050.

[10] Yin R, Kara EC, Li Y, DeForest N, Wang K, Yong T, et al. Quantifying flexibility of commercial and residential loads for demand response using setpoint changes. Appl Energy 2016;177:149-64. doi:10.1016/j.apenergy.2016.05.090.

[11] Vivekananthan C, Mishra Y, Ledwich G, Li F. Demand response for residential appliances via customer reward scheme. IEEE Trans Smart Grid 2014;5:809-20. doi:10.1109/TSG.2014.2298514.

[12] Safdarian A, Fotuhi-Firuzabad M, Lehtonen M. Benefits of Demand Response on Operation of Distribution Networks: A Case Study. Syst Journal, IEEE 2014;PP:1-9. doi:10.1109/JSYST.2013.2297792. 
[13] Sajjad IA, Chicco G, Napoli R. Definitions of Demand Flexibility for Aggregate Residential Loads. IEEE Trans Smart Grid 2016;7:2633-43. doi:10.1109/TSG.2016.2522961.

[14] Abiri-Jahromi A, Bouffard F. Contingency-Type Reserve Leveraged Through Aggregated Thermostatically-Controlled Loads Part I: Characterization and Control. IEEE Trans Power Syst 2016;31:1972-80. doi:10.1109/TPWRS.2015.2466175.

[15] Lu N, Chassin DP. A State-Queueing Model of Thermostatically Controlled Appliances. IEEE Trans Power Syst 2004;19:1666-73. doi:10.1109/TPWRS.2004.831700.

[16] Korkas CD, Baldi S, Michailidis I, Kosmatopoulos EB. Occupancy-based demand response and thermal comfort optimization in microgrids with renewable energy sources and energy storage. Appl Energy 2016;163:93-104. doi:10.1016/j.apenergy.2015.10.140.

[17] Chassin DP, Rondeau D. Aggregate modeling of fast-acting demand response and control under real-time pricing. Appl Energy 2016;181:288-98. doi:10.1016/j.apenergy.2016.08.071.

[18] Chassin DP, Stoustrup J, Agathoklis P, Djilali N. A new thermostat for real-time price demand response: Cost, comfort and energy impacts of discrete-time control without deadband. Appl Energy 2015;155:816-25. doi:10.1016/j.apenergy.2015.06.048.

[19] D'hulst R, Labeeuw W, Beusen B, Claessens S, Deconinck G, Vanthournout K. Demand response flexibility and flexibility potential of residential smart appliances: Experiences from large pilot test in Belgium. Appl Energy 2015;155:79-90. doi:10.1016/j.apenergy.2015.05.101.

[20] Waite M, Modi V. Potential for increased wind-generated electricity utilization using heat pumps in urban areas. Appl Energy 2014;135:634-42. doi:10.1016/j.apenergy.2014.04.059.

[21] Li P-H, Pye S. Assessing the benefits of demand-side flexibility in residential and transport sectors from an integrated energy systems perspective. Appl Energy 2018;228:965-79. doi:10.1016/j.apenergy.2018.06.153.

[22] Green R. The Effects of Cycling on Heat Pump Performance. Chester: 2012.

[23] Good N, Zhang L, Navarro-Espinosa A, Mancarella P. High resolution modelling of multi-energy domestic demand profiles. Appl Energy 2015;137:193-210. doi:10.1016/j.apenergy.2014.10.028.

[24] Mancarella P, Chicco G. Real-Time Demand Response From Energy Shifting in Distributed MultiGeneration. IEEE Trans Smart Grid 2013;4:1928-38. doi:10.1109/TSG.2013.2258413.

[25] Richardson I, Thomson M, Infield D, Clifford C. Domestic electricity use: A high-resolution energy demand model. Energy Build 2010;42:1878-87. doi:10.1016/j.enbuild.2010.05.023.

[26] McKenna E, Thomson M. High-resolution stochastic integrated thermal-electrical domestic demand model. Appl Energy 2016;165:445-61. doi:10.1016/j.apenergy.2015.12.089.

[27] Dimplex renewables. Dimplex heat pump. 2012.

[28] Wang W, Xiao J, Guo QC, Lu WP, Feng YC. Field test investigation of the characteristics for the air source heat pump under two typical mal-defrost phenomena. Appl Energy 2011;88:447080. doi:10.1016/j.apenergy.2011.05.047.

[29] National Grid. STOR general descrition of the service. 2015.

[30] National Grid. SHORT TERM OPERATING RESERVE ANNUAL MARKET REPORT 2012 / 13. 2013. 
[31] Department of Energy \& Climate Change. RHI_monthly_official_statistics_tables_31_December_2015 2016.

[32] Chartered Institute of Building Services Engineers. Environmental Design: CIBSE guide A. 7, illustr ed. CIBSE; 2006.

[33] Richardson I, Thomson M. Integrated simulation of photovoltaic micro-generation and domestic electricity demand: a one-minute resolution open-source model. Proc Inst Mech Eng Part A J Power Energy 2012;227:73-81. doi:10.1177/0957650912454989.

[34] Good N, Zhang L, Navarro-Espinosa A, Mancarella P. High resolution modelling of multi-energy domestic demand profiles. Appl Energy 2015;137. doi:10.1016/j.apenergy.2014.10.028.

[35] Chapman N, Zhang L, Good N, Mancarella P. Exploring flexibility of aggregated residential electric heat pumps. 2016 IEEE Int. Energy Conf., IEEE; 2016, p. 1-6. doi:10.1109/ENERGYCON.2016.7514082.

[36] National Grid. Short Term Operating Reserve - General Description of the Service 2017. doi:10.1017/CBO9781107415324.004. 